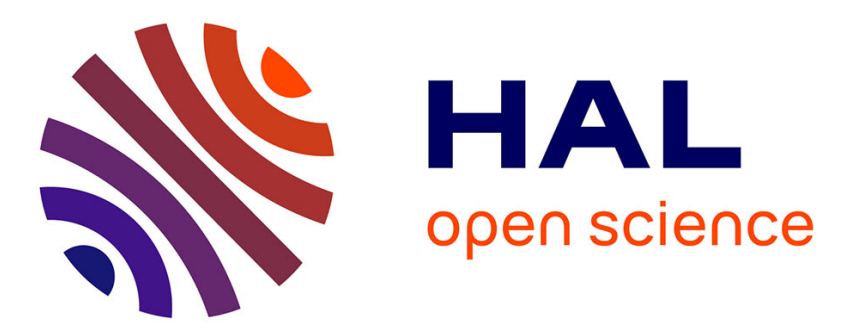

\title{
Livrer en circuits courts : les mobilités des agriculteurs comme révélateur des territoires alimentaires émergents. Étude de cas dans les Hauts-de-France
}

\author{
Gwenaelle Raton, Céline Raimbert
}

\section{- To cite this version: \\ Gwenaelle Raton, Céline Raimbert. Livrer en circuits courts: les mobilités des agriculteurs comme révélateur des territoires alimentaires émergents. Étude de cas dans les Hauts-de-France. Géocar- refour, 2020, 93 (3), 23p. 10.4000/geocarrefour.13993 . hal-03143901}

\author{
HAL Id: hal-03143901 \\ https://hal.science/hal-03143901
}

Submitted on 17 Feb 2021

HAL is a multi-disciplinary open access archive for the deposit and dissemination of scientific research documents, whether they are published or not. The documents may come from teaching and research institutions in France or abroad, or from public or private research centers.
L'archive ouverte pluridisciplinaire HAL, est destinée au dépôt et à la diffusion de documents scientifiques de niveau recherche, publiés ou non, émanant des établissements d'enseignement et de recherche français ou étrangers, des laboratoires publics ou privés. 


\section{Livrer en circuits courts : les mobilités des agriculteurs comme révélateur des territoires alimentaires émergents. Étude de cas dans les Hauts-de-France.}

Gwenaëlle Raton, chargée de recherche, IFSTTAR/AME/SPLOTT, gwenaelle.raton@ifsttar.fr

Céline Raimbert, post-doctorante, IFSTTAR/AME/SPLOTT, celine.raimbert@gmail.com

$* * *$ Remerciements :

Les auteures tiennent à remercier les partenaires du projet COLCICCA (CEREMA et Chambre d'Agriculture du Nord - Pas de Calais) et son financeur, la région Hauts-de-France ainsi que les producteurs et productrices enquêté·e·s dans ce cadre. Elles remercient également les relecteur-rice·s de ce texte pour leurs remarques et conseils avisés.

Résumé

Dans un contexte de reconnexion entre agriculture et alimentation, de plus en plus de producteurs agricoles se muent en acteurs du commerce alimentaire. C'est le cas de ceux qui commercialisent leur production en circuits courts alimentaire de proximité (CCAP), ce qui les conduit à assurer de nouvelles tâches telles que la livraison. Pour le géographe, il ne s'agit plus d'observer l'aire de chalandise d'un commerce, mais des pratiques de mobilité d'acteurs reliant l'espace productif à des bassins de consommation. Que disent ces mobilités sur les liens construits/en construction avec les divers espaces voisins des fermes ? Cet article propose d'interroger les mobilités des producteurs et d'analyser les territoires construits par ces mobilités. A partir de l'étude de six producteurs du Nord, il s'agit de qualifier des territoires de mobilité au regard des aires d'influence " classiques" des aires urbaines, mais aussi de bassins de production comme lieux de concentration au potentiel attractif ou répulsif. L'analyse détaillée des pratiques spatiales, en fonction des fréquences de livraison notamment, met en évidence des territoires de proximité subie ou choisie.

Mots-Clés: Circuits Courts Alimentaires de Proximité (CCAP); Mobilités; Représentations territoriales; Influence des aires urbaines ; Bassins de consommation/bassins de production ; Proximités géographiques.

\section{Abstract}

In a context of reconnection between agriculture and food, more and more producers are becoming actors of food supply. This is particularly the case of those who sell their production in local short food supply chain (SFSC), involving new tasks such as delivery. For the geographer, an analysis through the concept of catchment area becomes inoperative. It appears more relevant to focus on the mobility practices of the actors of the productive space towards consumers' areas. What these mobility practices can tell about the consolidated or under construction ties with the diverse spaces around the farms? This paper analyzes the farmers' mobility and the territories constructed by these practices. Thanks to a case study based on 6 farmers in the north of France, we aim to qualify their activity spaces depending on: first, the influence of urban areas, second, the influence of production areas considered as places of competition among producers, with attractive or repulsive effects. Our analysis of spatial practices of SFSC farmers, depending on the delivery frequencies for instance, also highlights mechanisms of suffered or chosen proximities.

Key words: Short food supply chain (SFSC); Mobility practices; Spatial representations; Influence of urban areas; Consumers'/Producers' Areas; Proximity.

\section{Introduction}

Dans un contexte de promotion de la multifonctionnalité de l'agriculture (Hervieu, 2002), les mondes ruraux et le métier d'agriculteur connaissent des mutations profondes et renouvelées (Perrier-Cornet, 2017). Les 
changements structurels de l'activité agricole ont conduit à des évolutions majeures de l'organisation des activités agroalimentaires, mais aussi de la distribution alimentaire (Torre, Filippi, 2005) qui incitent à porter le regard, au-delà du tissu productif, sur le tissu commercial et les liens noués avec celui-ci. Avec l'essor des circuits courts alimentaires de proximité (CCAP) dont les débouchés se multiplient et se diversifient, et dans le cadre d'une reconnexion entre agriculture et alimentation (Lamine, Chiffoleau, 2012), de plus en plus de producteurs agricoles se muent en acteurs du commerce et de la distribution alimentaire. En réduisant le nombre d'intermédiaires, des tâches commerciales et logistiques échoient aux producteurs. Elles impliquent de développer des relations avec une clientèle potentielle ou constituée, mais aussi, bien souvent, de livrer. Dès lors, il ne s'agit plus d'observer l'aire de chalandise d'un commerce mais des pratiques de mobilité d'acteurs de l'espace productif vers des bassins de consommation, si bien que la focale du géographe est modifiée. Cela suppose d'interroger les processus à l'œuvre dans le choix des espaces livrés et, dans la mesure où le champ des lieux possibles est vaste, les représentations spatiales des producteurs motivant ces choix. Que disent les mobilités des producteurs en CCAP sur les liens construits/en construction avec les divers espaces contigus aux fermes ? Quels sont, du point de vue de ces pratiques de mobilités, les territoires émergents ? Quelle est leur taille, leur forme et leur orientation?

À partir de l'étude de cas de six producteurs en CCAP du Grand Hainaut (département du Nord), nous qualifierons les territoires construits par leurs mobilités au regard des aires d'influence " classiques » des aires urbaines (AU) mais aussi de bassins de production. Dans un premier temps, la démarche des auteures est discutée au regard de la littérature et des différentes méthodes utilisées pour caractériser les CCAP. Les producteurs enquêtés, le terrain d'étude et les méthodes d'analyse sont ensuite présentés. La troisième partie est consacrée à l'analyse de la diversité des territoires identifiés et des profils de producteurs correspondants. Le texte s'achève sur une discussion mettant en perspective la portée des cas. En croisant territoires de mobilités des agriculteurs et territoires alimentaires locaux en construction, nous ouvrons sur les enjeux des mobilités spatiales des agriculteurs et leurs conséquences sur les territoires et les systèmes alimentaires.

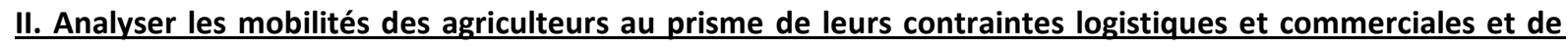 leurs représentations du territoire}

Afin de tenir compte de la diversité et de la complexité des CCAP, les auteures font le choix d'un objet d'analyse fin (les mobilités) qui est peu exploré dans la littérature sur les CCAP. Ces mobilités sont pensées en relation avec les territoires, dans leurs dimensions matérielles et idéelles. Cette approche répond à des questionnements théoriques et méthodologiques sur l'objet et l'échelle permettant de qualifier les spécificités de l'approvisionnement local et son inscription dans le territoire.

\section{Une diversité d'« objets » pour appréhender l'approvisionnement alimentaire}

S'intéresser à l'approvisionnement alimentaire des villes en géographie amène à faire un choix entre l'étude de bassins d'approvisionnement, l'étude filière ou encore l'étude de réseaux, notamment marchands. L'étude filière, si elle présente l'avantage de permettre l'analyse des stades successifs de production et de distribution d'un bien, induit une vision segmentée de l'approvisionnement par type de produit, qui semble de moins en moins valorisée dans la littérature actuelle, sauf pour des comparaisons circuits longs/chaînes courtes (Schlich et al., 2006 ; Coffi Dit Gleize, 2012).

Dans le cadre des CCAP, les travaux sur les bassins d'approvisionnement connaissent au contraire un regain d'intérêt, dans la mesure où ils permettraient de questionner la relocalisation de l'approvisionnement alimentaire (Baysse-Lainé, 2017). Néanmoins, le phénomène de relocalisation reste encore difficile à percevoir (Baysse-Lainé, Perrin, 2017) : est-ce lié au caractère récent du phénomène et à la difficulté de l'appréhender, quelle que soit l'échelle, ou à l'inefficience de l'échelle d'observation bassin d'approvisionnement ? Les travaux de l'écologie territoriale montrent que l'étude des bassins d'approvisionnement permet de rendre compte de l'évolution de la taille et de la forme des territoires alimentaires des villes et de mettre en évidence les bouleversements majeurs, dans les domaines agricoles, industriels et commerciaux, ayant conduit aux systèmes alimentaires contemporains (Bognon, 2014, Bognon et al., 2017). De telles recherches supposent un recul historique conséquent (Bognon, 2015), que les formes actuelles de CCAP (caractère récent et phénomène minoritaire en essor) ne semblent pas permettre.

Des transformations spatiales sont pourtant en cours et à défaut de pouvoir les lire à l'échelle des bassins d'approvisionnement, nous proposons de tenter de les observer à une échelle plus fine, celle des mobilités des 
acteurs. Avec l'essor des pratiques de livraison par les agriculteurs en CCAP, un changement majeur des mobilités des acteurs de l'approvisionnement s'est en effet déjà opéré en France. Dans le cadre de ces bassins d'approvisionnement local, il convient de comprendre les décisions commerciales individuelles d'exploitations agricoles, soit l'analyse des déterminants des mobilités des agriculteurs.

Ce type d'analyse revient à s'intéresser aux stratégies et pratiques d'individus. Cette démarche, assez courante dans les travaux sur les CCAP (Filippini et al., 2016), ne concerne pas, à notre connaissance, les mobilités des acteurs de la chaîne courte. Les travaux dans les pays du Sud apportent, pour leur part, des éléments mobilisables : d'une part, avec l'essor du vivrier marchand (Chaléard, 1996), les pratiques de nomadisme commercial (Allix, 1923), qui supposent un déplacement des producteurs des périphéries urbaines vers la ville ou des commerçants urbains vers les marchés périphériques, y ont été étudiées ; d'autre part, leur approche a permis la reconstitution de tout ou partie du bassin d'approvisionnement d'une ville, à partir des pratiques de mobilités des acteurs de l'approvisionnement et des choix des origines/destinations (Ninot 2003 ; Le Gall, 2011 ; Raton 2012). S'inspirant de ces travaux qui valorisent l'approche réseau, nous faisons le choix d'appréhender les CCAP par les mobilités des producteurs et les stratégies qui déterminent l'orientation de leurs déplacements, sans aller toutefois jusqu'à la reconstitution de bassins d'approvisionnement entiers. Focalisant notre attention sur les individus plus que sur les flux de marchandises, nous utilisons le terme de " mobilité » à la place de "circulation » et définissons la mobilité par le déplacement et par l'espace produit par ce déplacement (Lévy, Lussault, 2003 ; Lombard, Ninot, 2010 ; Caron, Torre, 2005).

\section{Une démarche propre à l'analyse des mobilités des agriculteurs en CCAP et à leurs enjeux logistiques}

Les mobilités des acteurs de la production en CCAP ne peuvent s'appréhender comme celles des consommateurs ou des acteurs du commerce, ce qui conduirait à dessiner l'aire de chalandise d'un ou d'un ensemble de points de vente. Notre objectif est autre : comprendre les choix des lieux livrés en ville à partir de la localisation de la ferme. Nous nous inspirons, pour ce faire, des travaux de géographie et de sociologie du travail sur les mobilités des actifs ayant à se déplacer plusieurs fois par jour pour des raisons professionnelles. Bien que peu nombreux, ils permettent d'éclairer des mobilités professionnelles, notamment par métier (Belton \& De Conink, 2007) ou par territoire (Delmas, 2015, Aguilera et al., 2007 ; Gressel \& Munduteguy, 2008), pour envisager des «territoires d'interventions professionnelles » désignant à la fois l'adaptation des déplacements professionnels aux contraintes urbaines et la façon dont le territoire est modelé par eux (Bahoken et al., 2018). Dans la lignée de ces travaux, nous appréhendons la mobilité spatiale des agriculteurs en définissant des territoires de mobilité, dont les tailles et orientations seraient le résultat de stratégies commerciales et logistiques.

Les travaux sur les enjeux logistiques des chaînes courtes montrent que les opérations logistiques et de transport sont majoritairement réalisées par les producteurs de manière individuelle et interne à l'exploitation (Blanquart et al., 2015). Etant donné le temps conséquent consacré aux livraisons (en moyenne $8 \mathrm{~h}$ par semaine, Raton et al., 2018) et le poids des coûts logistiques sur la rentabilité des activités en CCAP dans les exploitations agricoles (Raton et al, 2015), les stratégies logistiques semblent jouer un rôle clé dans les choix des lieux de commercialisation et de livraison des producteurs agricoles et ainsi dans la construction de leur territoire de mobilité.

\section{$\underline{\text { Intégrer le territoire, la localisation et les représentations à l’analyse des mobilités }}$}

Plutôt que de chercher à comprendre les conséquences spatiales d'une relocalisation de l'approvisionnement alimentaire, nous centrons notre étude sur les territoires de mobilité, eux-mêmes fruits de décisions propres à chaque producteur. Le recours au concept de territoire vise à souligner la dimension duelle, à la fois matérielle et idéelle de ces objets, qui ne sont pas simplement des espaces de circulation, mais aussi des espaces appropriés (Di Méo, Buléon, 2005). En effet, les choix et pratiques de mobilités reposent à la fois sur des données objectives concernant les espaces habités et les espaces livrés (la distance entre la ferme et tel lieu, la taille de telle ville, etc.) et sur des construits subjectifs concernant les représentations que les producteurs se font de ces espaces. Ainsi, la prise en compte des représentations n'est pas un élément supplémentaire de caractérisation du territoire de mobilité mais une focale indispensable à la compréhension des stratégies d'acteurs.

En réinterrogeant les relations entre mondes ruraux et leur environnement ainsi que l'émergence de territoires sous l'effet de nouvelles pratiques agroalimentaires et de distribution, nous nous inscrivons dans la lignée des travaux sur les proximités. Les choix réalisés par les producteurs agricoles, en tant qu'acteurs économiques, semblent déterminés par des proximités géographiques. Ces dernières considèrent non seulement la distance 
physique entre deux objets mais aussi la distance relativisée par le capital de mobilité des individus et leur perception de la distance et de ses externalités (Pecqueur \& Zimmermann, 2004 ; Rallet \& Torre, 2004 ; Talbot \& Kirat, 2005). Y recourir permet de souligner le rôle des représentations des espaces habités et des espaces livrés sur les choix de mobilités. Elles peuvent ainsi influer sur les stratégies commerciales et logistiques en allongeant ou raccourcissant les distances objectives, notamment dans les relations ville/campagne. Nous posons l'hypothèse que, sans avoir à intégrer de manière précise les coûts et prix, les mobilités traduisent les arbitrages réalisés.

Les proximités s'entendent aussi de façon dynamique. Recherchées ou subies, elles se caractérisent par un potentiel d'interactions qui peut être ou non activé, au gré des stratégies et préférences des acteurs économiques (Torre, 2009). Or, pour l'étude de mobilités spatiales, l'intérêt d'une analyse par les représentations territoriales ne réside pas tant dans ces représentations elles-mêmes que dans les interactions ou les absences d'interactions qu'elles vont susciter entre les lieux.

\section{Matériel et méthode}

\section{1 Producteurs enquêtés}

L'analyse se base sur l'étude de six producteurs en CCAP, enquêtés dans le cadre du projet COLCICCA (Concevoir des Organisations Logistiques Collectives et Intelligentes pour les Circuits Courts Alimentaires). Plusieurs groupes de producteurs en recherche d'optimisation logistique ont été accompagnés par des acteurs territoriaux et des chercheurs. Les enquêtés appartiennent à l'association Les Paysans du Grand Hainaut, fondée en 2015, dans le but de livrer la restauration collective dans le Grand Hainaut ${ }^{1}$. Cet article utilise les données ayant servi au diagnostic logistique individuel.

Collectées entre octobre 2017 et mars 2018 lors d'entretiens semi-directifs, elles portent sur :

- Les caractéristiques générales de leur exploitation: profil productif, surface exploitée, type de structure ;

- Leur activité en CCAP : types de production, vision diachronique sur les stratégies mises en place (passé, présent, futur) ;

- Leur organisation logistique pour les débouchés en CCAP : liste exhaustive des débouchés livrés sur l'année écoulée, type de débouchés, localisation, véhicule utilisé, type de trajets (tournée ou trace directe), outils utilisés et temps consacrés aux tâches logistiques.

La posture d'accompagnement inhérente au projet a permis de suivre les producteurs sur la période 2017-18 et d'observer les évolutions dans leurs stratégies commerciales et logistiques. Les six producteurs présentent des profils variés, malgré une dominante de transformation laitière, reflet des orientations du Grand Hainaut (Tableau 1). Par la suite et dans le respect de l'anonymat, les producteur.rice·s sont identifié·e.s par des lettres et tou·te·s féminisé.e.s.

\footnotetext{
${ }^{1}$ Le collectif est soutenu par la DRAAF, le Département du Nord et la Chambre d'Agriculture et accompagné par l'ADARTH.
} 
Tableau 1 Profil des productrices enquêtées. Source : Enquêtes COLCICCA

\begin{tabular}{|c|c|c|c|c|c|c|}
\hline Productrice & A & B & C & D & E & F \\
\hline Profil productif & Maraîchage & $\begin{array}{c}\text { Polyculture- } \\
\text { élevage }\end{array}$ & $\begin{array}{c}\text { Polyculture- } \\
\text { élevage }\end{array}$ & $\begin{array}{c}\text { Polyculture- } \\
\text { élevage }\end{array}$ & $\begin{array}{c}\text { Polyculture- } \\
\text { élevage }\end{array}$ & $\begin{array}{c}\text { Polyculture- } \\
\text { élevage }\end{array}$ \\
\hline $\begin{array}{c}\text { Surface de } \\
\text { l'exploitation }\end{array}$ & 35 ha & 100 ha & 140 ha & 55 ha & 128 ha & 75 ha \\
\hline $\begin{array}{c}\text { Type de } \\
\text { structure }\end{array}$ & $\begin{array}{c}\text { Exploitation } \\
\text { individuelle }\end{array}$ & EARL & GAEC & $\begin{array}{c}\text { Etablissement } \\
\text { public }\end{array}$ & $\begin{array}{c}\text { Exploitation } \\
\text { individuelle + } \\
\text { SASU }\end{array}$ & GAEC \\
\hline $\begin{array}{c}\text { Année } \\
\text { d'installation }\end{array}$ & 1987 & 1995 & 2009 & 1976 & 2009 & 1992 \\
\hline $\begin{array}{c}\text { Produits vendus } \\
\text { en CCAP }\end{array}$ & Légumes & $\begin{array}{c}\text { Produits laitiers } \\
\text { \& Fraises, } \\
\text { Tomates }\end{array}$ & Produits laitiers & Produits laitiers & $\begin{array}{c}\text { Pommes de terre } \\
\text { et frites sous- } \\
\text { vide }\end{array}$ & $\begin{array}{c}\text { Produits } \\
\text { laitiers }\end{array}$ \\
\hline $\begin{array}{c}\text { Début de } \\
\text { l'activité CCAP }\end{array}$ & 1990 & 2009 & 2009 & 1992 & 2009 & 2009 \\
\hline $\begin{array}{c}\text { Proportion } \\
\text { activité CCAP }\end{array}$ & $70-80 \%$ & $20 \%$ & NSP & NSP & Inférieur à $50 \%$ & $40 \%$ \\
\hline
\end{tabular}

NSP = ne sait pas

\section{2 Terrain d'étude}

Outre le fait de participer à un même projet collectif, les productrices se situent sur un territoire commun : le Grand Hainaut (Carte 1). II est composé de 9 établissements publics de coopération intercommunale (EPCI) dont 4 communautés d'agglomération (CA) et 5 communautés de communes (CC). L'intérêt de ce territoire réside dans son caractère multipolarisé, entre deux villes ou bassins de consommations situés à égales distances, d'où des possibilités de choix pour les producteurs.

\section{Carte 1 Le Grand Hainaut et les productrices enquêtées}

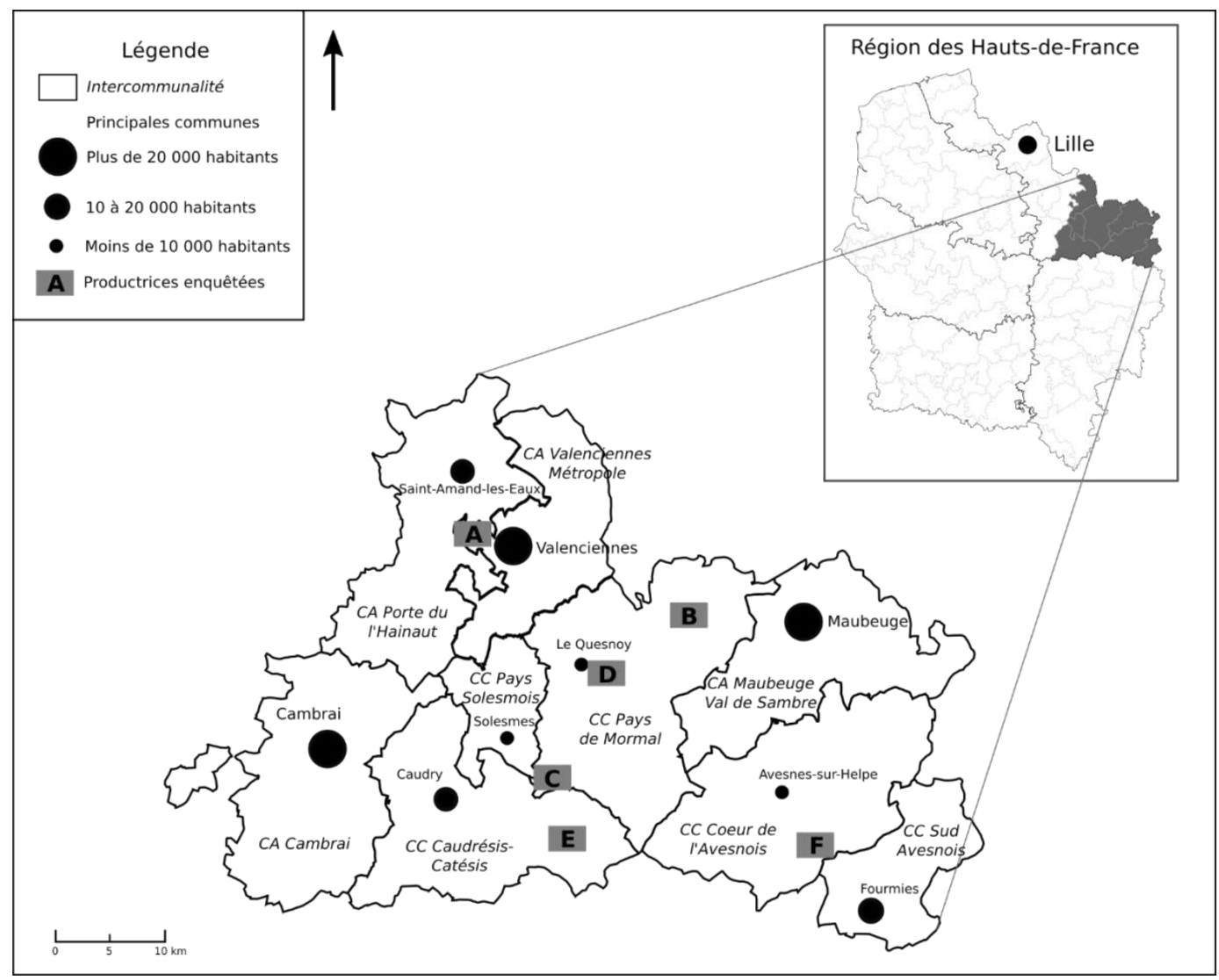


Le Grand Hainaut s'étend sur la moitié sud du département du Nord, il en est sa portion la plus rurale (262 hab./ $\mathrm{km}^{2}$ contre 453 à l'échelle du département). Il compte néanmoins plusieurs grands pôles urbains: Valenciennes, Maubeuge, Cambrai et, dans une moindre mesure, Saint-Amand-les-Eaux. Ce territoire est également la partie la moins dynamique d'un point de vue économique et social, avec des chiffres en-deçà de la moyenne départementale : un revenu médian de $17691 €$ contre $19920 €$, un taux de pauvreté de 22,8\% contre $19,4 \%$, etc. (INSEE, 2015). Les principaux foyers de fragilité sociale sont localisés dans les secteurs anciennement industriels de Maubeuge et de Fourmies et dans les espaces ruraux du Cambrésis (CA Cambrai et CC Caudrésis-Catésis). Outre une orientation industrielle marquée (en moyenne, une part de 6,4\% contre 4,9\% dans le département du $\operatorname{Nord}^{2}$ ), l'agriculture est également surreprésentée : 8,45\% contre 3,2\% ${ }^{3}$. Au total, quatre unités agricoles homogènes sont présentes dans le Grand Hainaut (Carte 2) (Agreste, 2012; SCoT Sambre-Avesnois, 2017).

\section{Carte 2 L'orientation technico-économique dominante du Grand Hainaut}

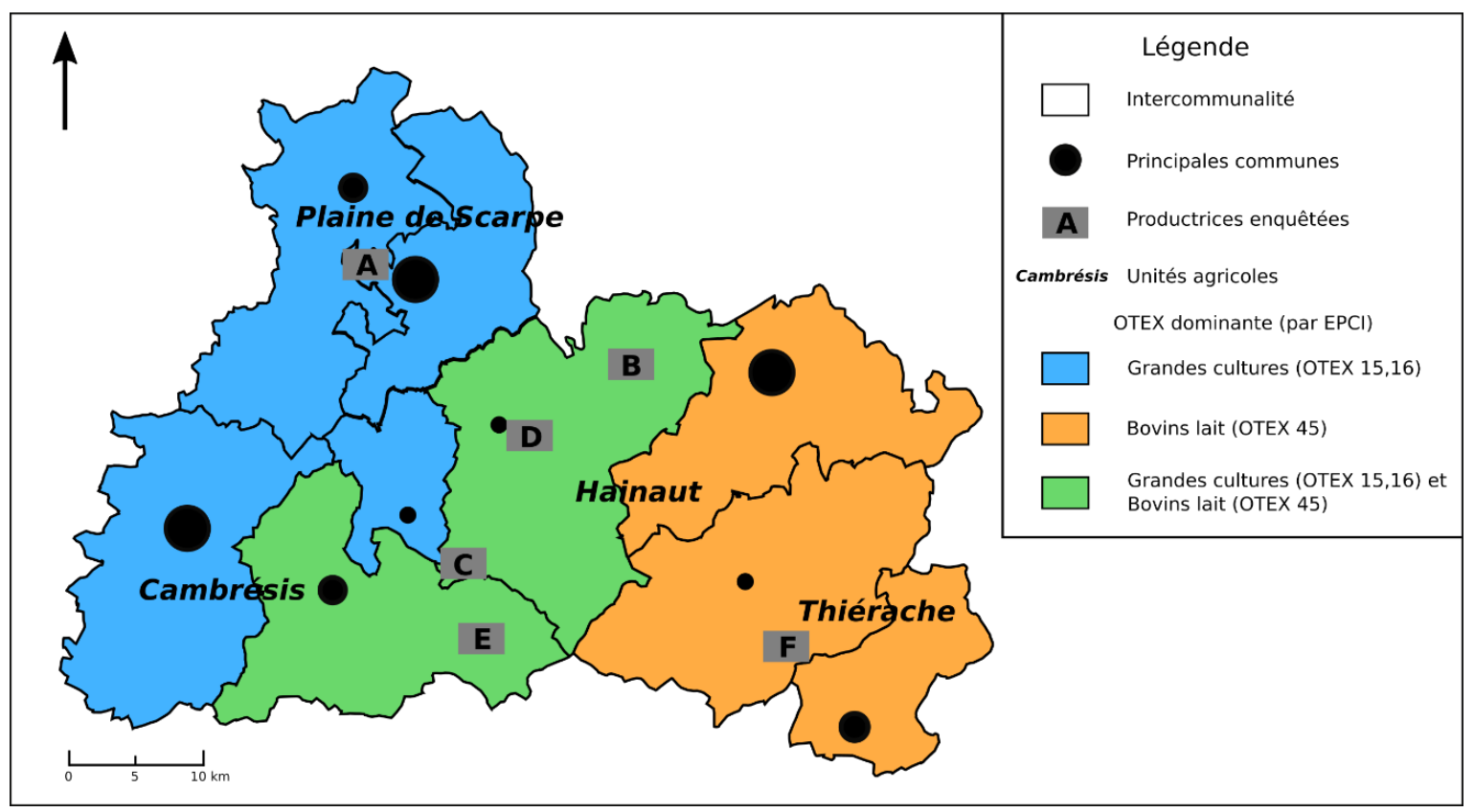

Conception : Céline Raimbert. Source : Agreste, 2010.

\section{III.3 Caractérisation des territoires de mobilité}

\section{Données mobilisées pour caractériser les territoires de mobilité}

Les territoires de mobilité de chaque individu sont dessinés à partir de trois principales données :

- Le point de départ, autrement dit la commune où est localisée l'exploitation ;

- Les points livrés par chaque productrice. Les débouchés en CCAP non livrés n'ont pas été pris en compte.

- Les caractéristiques du trajet de livraison : fréquence des livraisons et organisation logistique (tournée ou trace directe), cette dernière pouvant renseigner sur la sophistication des stratégies. En effet, sauf

\footnotetext{
${ }^{2}$ En nombre d'établissements actifs au 31 décembre (INSEE, 2015)

3 Idem

${ }^{4}$ L'ensemble des productrices vendent directement à la ferme, soit via un point de vente individuel (sont concernées A, B, C et D), soit parce que certains de leurs clients viennent eux-mêmes récupérer leurs commandes sur l'exploitation.
} 
exceptions de nature structurelle $e^{5}$, la tournée est identifiée comme l'une des premières étapes de l'optimisation logistique. L'analyse prend en compte l'ensemble des livraisons sur la période T - T-1 an ( $T$ = le mois et l'année de l'enquête) et leur fréquence. La période observée n'est pas strictement la même pour toutes les productrices, mais elle recouvre un laps de temps identique, permettant de limiter les effets de saisonnalité.

\section{Variables retenues pour formaliser ces territoires}

Trois familles d'indicateurs sont retenues :

1- Deux types de distance :

- Entre l'exploitation et les points livrés. Exprimées en kilomètres, elles ont été calculées « par la route " sous Google Map. Elles ne reflètent pas nécessairement le nombre réel de kilomètres parcourus mais permettent d'évaluer l'éloignement à la ferme.

- Entre l'exploitation et le centre moyen des points livrés. Les centres moyens ont été calculés sous ArcGis et représentent le centre de concentration d'un ensemble d'entités qui sont les points livrés de chaque exploitation. Afin de prendre en compte la fréquence des points livrés, le centre moyen pondéré à la fréquence des livraisons a également été calculé. Toutes les données détaillées sur les distances et profils figurent en annexe.

2- Les ellipsoïdes ou ellipses d'écart-type récapitulent les caractéristiques spatiales de l'espace de mobilité : tendance centrale, dispersion et tendances directionnelles. Elles sont utilisées pour identifier la forme et l'empreinte spatiale des territoires de mobilité. Les ellipsoïdes ont également été pondérés. L'analyse s'appuie sur la comparaison des formes avec et sans pondération.

3- Deux unités territoriales complémentaires sont mobilisées selon le profil des productrices :

- Les AU d'appartenance de l'exploitation et des points livrés. Fondées sur l'identification de zones d'emploi et de déplacements domicile-travail, ce sont neuf unités homogènes mettant en évidence des aires d'influence et des effets de polarité, au-delà des limites physiques des villes (INSEE, 2015c). Cette échelle a pour intérêt de tester l'hypothèse selon laquelle les stratégies des productrices suivraient les logiques d'aires d'influence des villes.

- Les EPCl d'appartenance de l'exploitation et des points livrés. Il s'agit d'une unité territoriale intermédiaire fournissant des indications sur le périmètre des territoires de mobilité, mais aussi des caractéristiques socio-économiques des espaces fréquentés ou évités, ce qui n'est pas possible avec l'approche par les AU.

Enfin, l'ensemble des données chiffrées (voir annexes) sont pondérées par la fréquence des livraisons : chaque point livré ne vaut pas 1 , mais x (x étant le nombre de fois où il a été livré sur une année). Cette pondération permet de ne pas surreprésenter certains débouchés qui ne sont livrés que très occasionnellement et de rendre plus précisément compte de l'intensité des déplacements.

\section{Résultats}

L'analyse détaillée des mobilités de six productrices met en évidence trois types de stratégies aboutissant à la construction de territoires de mobilité. 1. sollicitation de territoires d'appartenance quels qu'ils soient; 2 . mobilisation de la proximité de foyers de population de densités variables; et 3 . prise en compte des caractéristiques des bassins de production comme éléments d'attraction/répulsion. Ces stratégies plus ou moins combinées donnent lieu à des territoires dont l'orientation et la forme varient, qui sont plus ou moins concentrés, polarisés et dont l'empreinte spatiale intègre une plus ou moins grande diversité d'espaces.

\footnotetext{
${ }^{5}$ Certains débouchés ou conditions de livraisons peuvent rendre difficile l'organisation de tournées : temps de présence sur les marchés ou AMAP, volumes transportés (Gonçalves, 2013 ; Raton et al., 2018).
} 


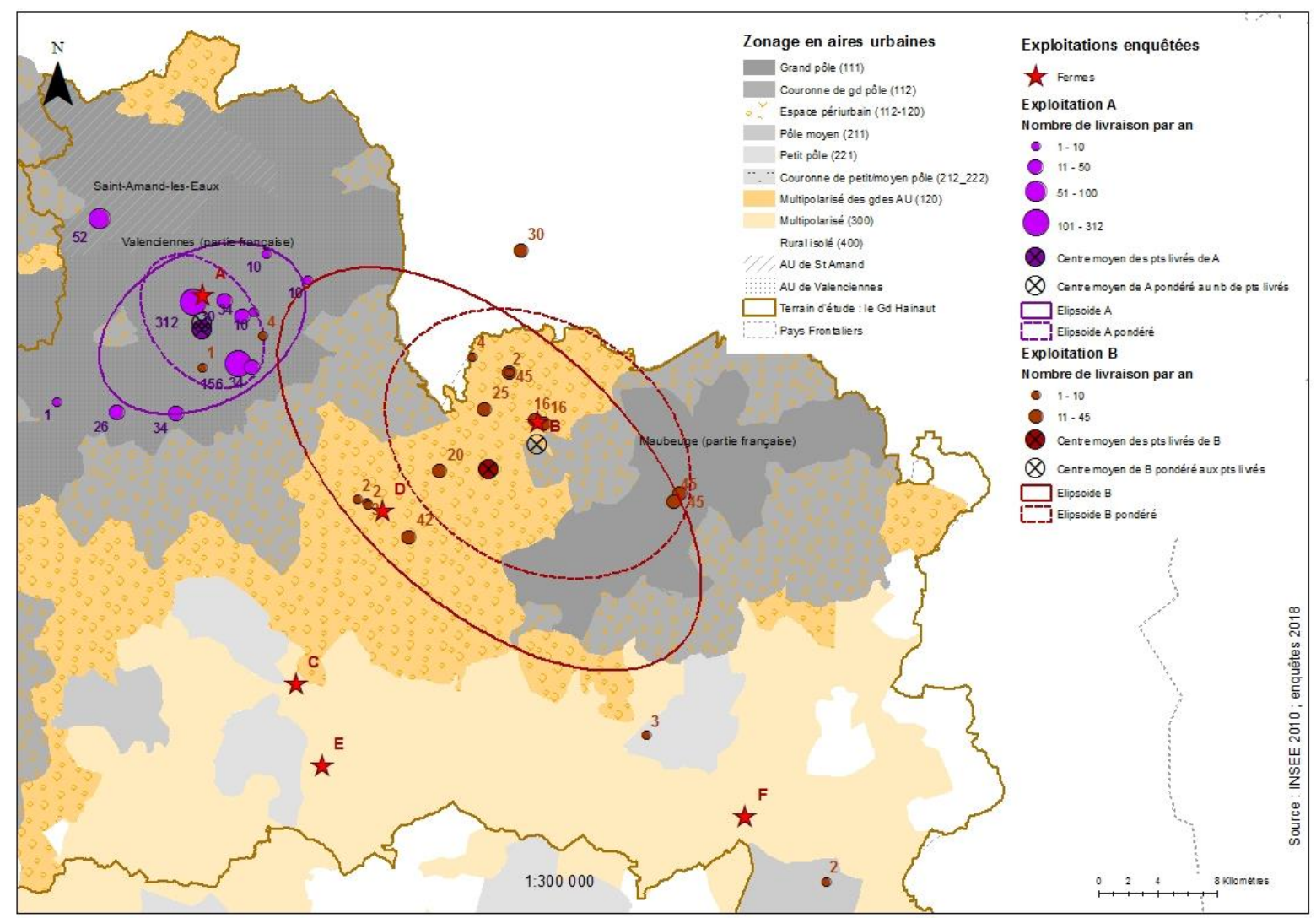

Conception : Gwenaëlle Raton, IFSTTAR. Sources : INSEE, 2010 et enquêtes COLCICCA

\section{Le cas de A : un territoire très concentré et polarisé}

La productrice $A$ est maraîchère. Bénéficiant d'une localisation au cœur de l'AU de Valenciennes, elle privilégie les livraisons dans son territoire d'appartenance (Carte 3). Sa stratégie de mobilité se caractérise par une exclusivité spatiale, exprimée à des degrés divers, selon l'échelle considérée : tous les débouchés livrés se situent dans l'AU d'appartenance, à l'exception d'un point, et concentrés dans son pôle (93\% des livraisons). Ceci souligne un tropisme métropolitain fort, qui est le résultat de la proximité immédiate de la ferme avec un bassin de consommation très peuplé et relativement dynamique d'un point de vue économique ${ }^{6}$. Le territoire de mobilité de $\mathrm{A}$ est le plus concentré de l'ensemble du corpus. Elle présente la distance médiane (5 $\mathrm{km})$ et la distance maximale $(20 \mathrm{~km})$ parcourues les plus faibles. En outre, le centre moyen des points livrés est situé à seulement 2,2 km de la ferme, abaissé à 1,8 km lorsqu'on pondère à la fréquence des livraisons (voir annexes).

Cette logique de concentration se traduit aussi par un très faible nombre de points desservis (une dizaine) et une très faible diversité de débouchés (près de 70\% des livraisons sont à destination de la $\mathrm{GMS}^{7}$ ). Ceci influence également l'organisation logistique de la productrice. Malgré une réflexion logistique avancée (conditions de livraison, arrêt de livraison si le point n'est plus sur un trajet, etc.), les traces directes restent majoritaires (60\% des trajets) et concernent le supermarché, débouché historique, situé à moins d'1 km de l'exploitation.

\footnotetext{
${ }^{6}$ Bien qu'au sein du département du Nord Valenciennes affiche des scores économiques assez moyens (légèrement endeçà de la moyenne départementale), à l'échelle du Grand Hainaut, le bassin se positionne, avec ses $17087 €$ de revenu médian (INSEE, 2015) à la troisième place derrière Saint-Amand-les-Eaux et Cambrai.

${ }^{7}$ Grandes et moyennes surfaces.
} 
L'hyper-concentration du territoire de mobilité n'est pas seulement permise par la localisation de l'exploitation mais se couple à une stratégie commerciale fondée sur une proximité recherchée. Ce territoire est le fruit d'un lent processus de resserrement de ses débouchés autour de son exploitation depuis ses débuts dans les CCAP dans les années 1990. Cette stratégie a consisté en l'abandon progressif des clients jugés trop éloignés (de l'exploitation et/ou des points livrés) et une volonté clairement affirmée de ne pas livrer, sauf exception, hors du secteur de Valenciennes. Ainsi, A prévoit d'arrêter le seul point situé dans une autre AU, jugé trop lointain et trop coûteux. Cette modification de son organisation logistique semble le point d'aboutissement de sa stratégie de resserrement. En outre, sa situation géographique lui permet de se positionner favorablement dans son bassin de production : les agriculteurs exploitants représentent seulement $0,3 \%$ de la population active de l'AU de Valenciennes (INSEE, 2015), ce qui incite encore A à concentrer ses livraisons dans un secteur perçu comme peu concurrentiel.

$\underline{\text { Le cas de B : un territoire de concentration modérée et une diversification des pôles }}$

La productrice B débute dans les CCAP. Elle vend des fruits et légumes et des produits laitiers. Localisée dans I'aire d'influence de grands pôles, mais dans un espace multipolarisé (Carte 3), elle bénéficie d'une rente de localisation rendant accessibles deux bassins de consommation (Maubeuge et Valenciennes). Pourtant, ses livraisons se concentrent dans le périurbain (64\%), a priori moins dense. B n'exclut pas pour autant les grands pôles de Maubeuge et Valenciennes (34\%). La répartition des livraisons entre ces deux grands pôles suit une logique de proximité : Maubeuge, le grand pôle le plus proche est très nettement privilégié $(32,5 \%$ contre $2 \%$ pour Valenciennes). Tout comme A, B livre préférentiellement à proximité immédiate de son exploitation. La distance médiane parcourue demeure faible $(7 \mathrm{~km})$, la distance entre la ferme et le centre moyen des points livrés aussi $(4,5 \mathrm{~km}$, abaissée à $1,5 \mathrm{~km}$ avec pondération). Néanmoins, son territoire se révèle moins concentré, plus étendu et plus divers. La distance maximale parcourue est plus importante $(51 \mathrm{~km})$ et ce sont $6 \mathrm{EPCl}$ différents ainsi qu'une commune belge qui sont livrés. Plus de la moitié des livraisons (64\%) se fait à destination de l'EPCI d'appartenance de la productrice mais les espaces fréquentés sont tout aussi bien des grands pôles, leurs couronnes, que des petits ou moyens pôles. En dépit de la proximité de la Belgique, elle est la seule à franchir la frontière. On pourrait y voir une proximité géographique recherchée liée à une proximité sociale, dans la mesure où cette productrice, elle-même belge, livre en Belgique un producteur qu'elle connaît de longue date. À noter que ce déplacement a un double intérêt : livrer et s'approvisionner, donnant à cette mobilité un caractère d'optimisation mais aussi de réciprocité dans le sens donné à l'échange.

Le caractère parsemé des points de livraison peut s'expliquer par l'ouverture récente de l'exploitation aux CCAP et une stratégie encore peu consolidée, mais qui tend à se dessiner, pour se centrer autour du développement d'un point de vente propre : le magasin. B vient de le transférer de la ferme vers le centre-ville de sa commune de résidence. Dans ce cas, c'est davantage une logique de bassin de production qui peut être soulignée, la concentration de l'offre agricole rendant attractive le déplacement des consommateurs. Par ailleurs, B livre assez peu (307 livraisons sur l'année écoulée) et compte peu de clients : 19 au total, dont seuls 9 sont livrés régulièrement. Cela se traduit par une organisation logistique faiblement élaborée : les traces directes sont majoritaires (55\% des trajets) et les tournées irrégulières (composition différente, jours variables).

Finalement, les territoires de mobilité de A et B se révèlent comparables en termes de concentration. Pourtant, ces deux agricultrices présentent des profils productifs et commerciaux bien distincts ( $80 \%$ de l'activité en CCAP et stratégie consolidée pour A contre à peine $20 \%$ et stratégie en cours d'élaboration pour B). Dès lors, ce qui semble les réunir est la localisation de leur ferme qu'elles exploitent largement. La comparaison des formes pondérée et non pondérées des ellipsoïdes illustre ce phénomène : ce sont les deux seuls cas pour lesquels, la forme de l'ellipsoïde change lorsqu'il est pondéré. L'orientation du territoire est alors moins marquée et l'ellipsoïde tend à former un cercle concentrique dont la ferme est quasiment le point central.

\section{IV.2 Cas F et E : s'adapter aux spécificités de son territoire d'appartenance}

Carte 4 : Les territoires de mobilité de F et $E$ 


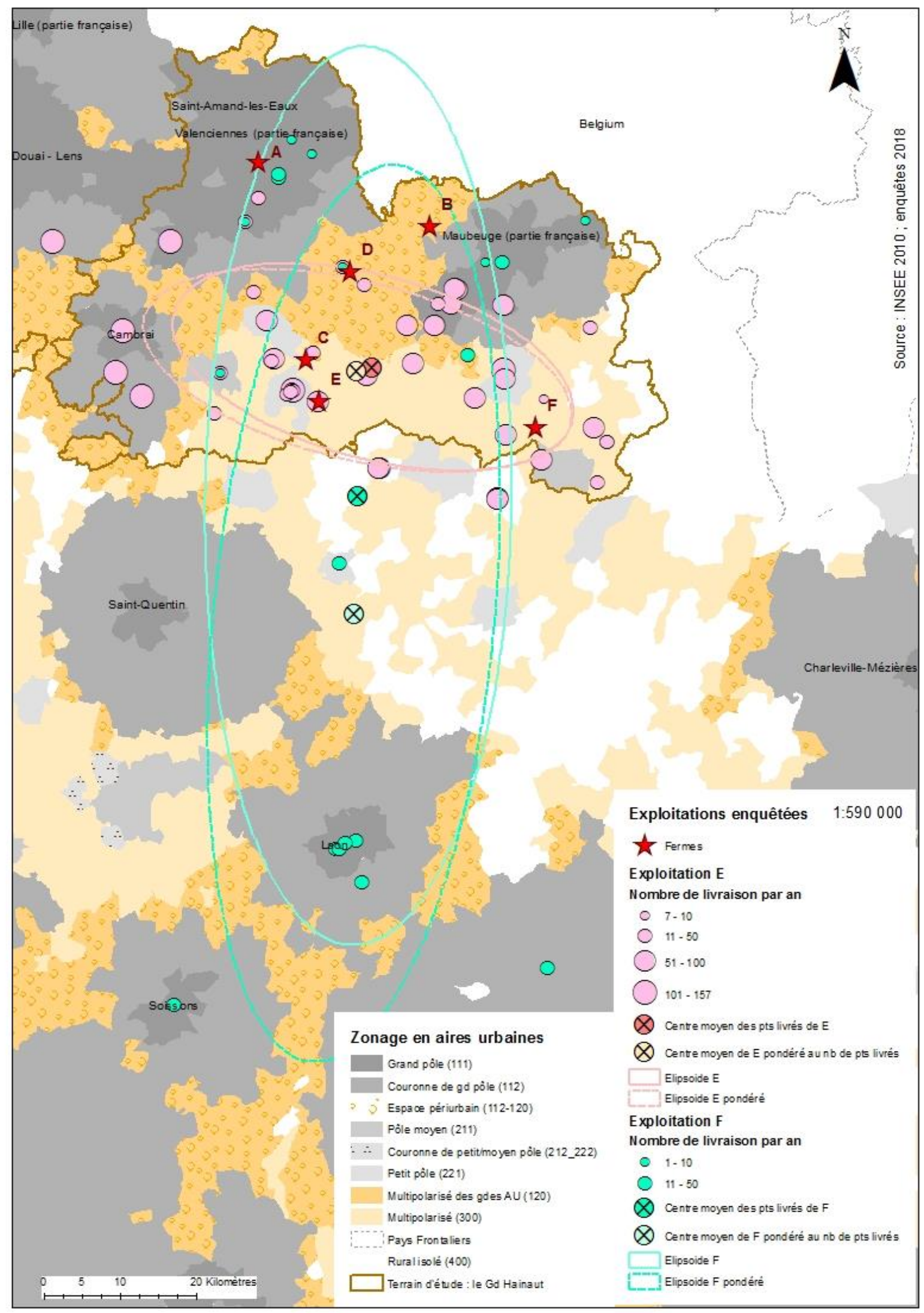

Conception : Gwenaëlle Raton IFSTTAR. Sources : INSEE, 2010 et enquêtes COLCICCA

$\underline{\text { Le cas de } \mathrm{F} \text { : un vaste territoire construit sur un tropisme urbain extraverti et dispersé }}$ 
Localisée dans le sud de l'Avesnois, $\mathrm{F}$ vend des produits laitiers transformés à la ferme. Cette productrice montre la volonté de s'extraire du territoire d'appartenance pour lui préférer d'autres bassins de consommation et de production. La part des CCAP dans le chiffre d'affaires global de son exploitation ne cesse de croître depuis les années 2000, suite aux crises successives du lait en Belgique et en France.

Parmi le corpus, F est celle qui parcourt les plus grandes distances. L'éloignement du territoire d'appartenance est lisible à l'observation des distances entre ferme et centre moyen des points livrés : $25 \mathrm{~km}$ et $34 \mathrm{~km}$ avec pondération (Carte 4). L'extraversion est aussi soulignée par la faible proportion de livraisons dans le territoire d'appartenance, à l'échelle de l'EPCI de la ferme (12\% des livraisons), mais aussi à celle du Grand Hainaut : sur les $10 \mathrm{EPCl}$ livrés par F, les 4 localisés en dehors du Grand Hainaut représentent $59 \%$ des livraisons. Cette proximité géographique subie est aussi exprimée par la distance minimale parcourue par $F$, bien supérieure à celle des autres productrices ( $16 \mathrm{~km}$ contre $1,4 \mathrm{~km}$ en moyenne) et par la position de l'ellipsoïde. C'est la seule productrice dont la ferme n'appartient pas à l'ellipsoïde, qu'elle soit pondérée ou non. Ainsi, en plus d'être extraverti, le territoire de mobilité de $\mathrm{F}$ se révèle discontinu.

Les mobilités de $\mathrm{F}$ soulignent un tropisme urbain certain : les livraisons à destination des grandes AU et de leur couronne sont très largement majoritaires (94\% du total). Au sein de celles-ci, les grands pôles sont les plus livrés : Laon (30\%), Valenciennes (17,5\%), Soissons (12\%) puis Maubeuge (10,5\%). Si F fréquente avant tout les grandes villes, ce ne sont ni les bassins de consommation les plus proches (Maubeuge), ni les plus densément peuplés qui sont recherchés. On observe ainsi un attrait spécifique de $\mathrm{F}$ pour les AU de l'Aisne (moins peuplées que celles du Nord) où la productrice se rend plusieurs fois par semaine. Cette préférence révèle surtout une répulsion vis-à-vis du territoire d'appartenance perçu négativement : selon $F$, la ruralité et la paupérisation de I'Avesnois et de la Thiérache ( $F$ réside à proximité du secteur de Fourmies, l'un des plus pauvres du Grand Hainaut) en font un bassin de consommation peu attractif, l'incitant à se tourner vers un bassin bien différencié. À ce titre, l'Aisne présente plusieurs avantages comparatifs, en termes de bassins de consommation (une population globalement plus aisée que les espaces à proximité immédiate de l'exploitation, Carte 5), et de bassins de production (territoire moins concurrentiel, l'Aisne étant plus céréalière que laitière, carte 2 ).

Carte 5 : La répartition des livraisons de F au regard du dynamisme socio-économique des EPCI

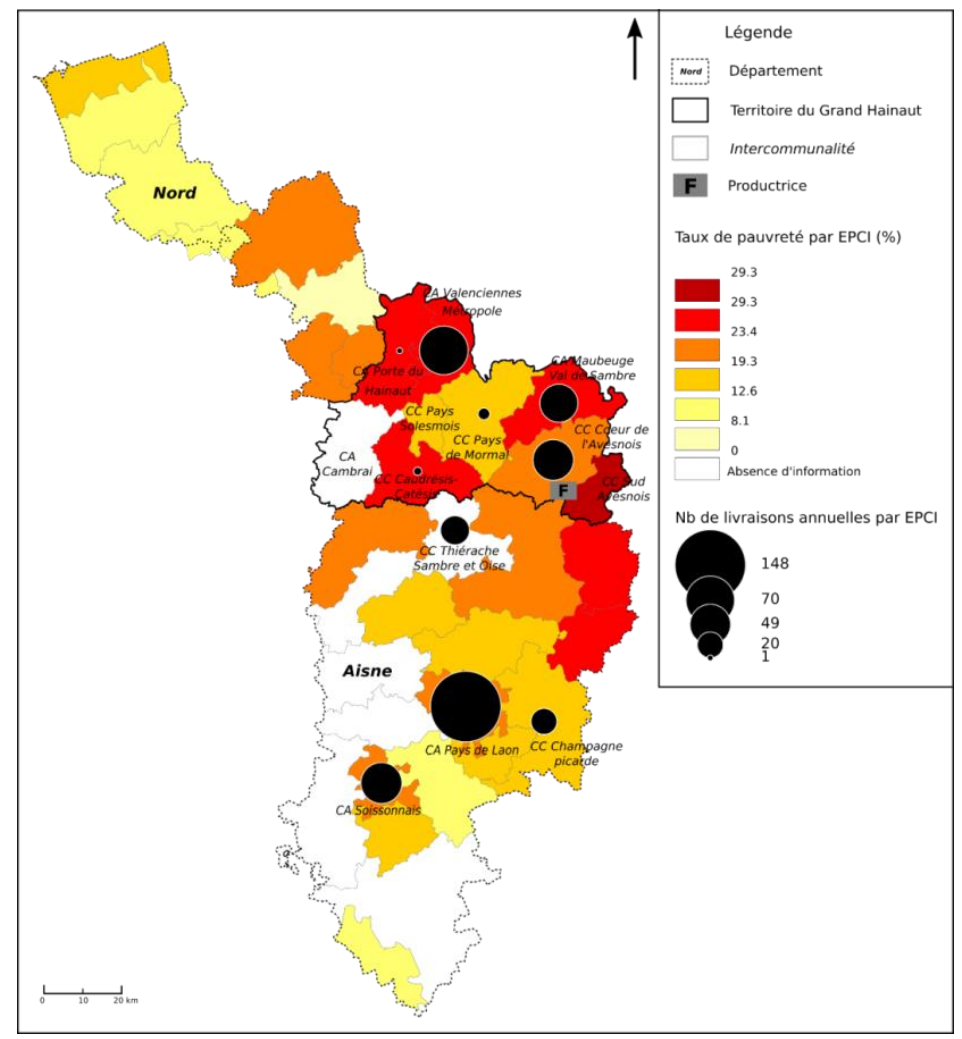


En outre, cette orientation vers l'Aisne, ancienne, se renforce. Le débouché le plus récent de F est à Soissons, qui est le lieu livré le plus éloigné de la ferme. Dans un contexte d'évolution de l'exploitation (départ en retraite de l'un des partenaires du GAEC), la productrice est en train de repenser sa stratégie commerciale. Elle souhaite se tourner encore davantage vers le territoire de l'Aisne, en même temps qu'elle abandonne certains débouchés plus proches de l'exploitation (moins de $10 \mathrm{~km}$ ) mais considérés comme peu ou plus rentables (ventes en tournée auprès d'une clientèle d'habitués, vieillissante et paupérisée). Cet argument a été avancé explicitement par la productrice pour justifier son orientation. Orientation qui lui permet de renouveler sa clientèle de particuliers en recourant à des modes de commercialisation plus novateurs (un drive fermier et une AMAP).

$\underline{\text { Le cas de } \mathrm{E} \text { : un territoire de mobilité relativement concentré mobilisant prioritairement les marges }}$

E est une productrice en polyculture-élevage. Son activité en CCAP se concentre exclusivement sur la vente de pommes de terre sous-vides, notamment sous forme de frites, auprès de la restauration commerciale. Comme F, l'exploitation de la productrice E se situe à proximité immédiate de l'Aisne (Carte 4). Le territoire de mobilité révèle pourtant des stratégies bien différentes. Il se distingue par une orientation affirmée vers les marges des grands pôles (près de $40 \%$ des livraisons). E est même la seule productrice de l'échantillon à livrer des " communes isolées hors influence des pôles » ( $13 \%$ du total). Quant aux livraisons vers les grandes AU, ce sont les moins représentées (19\%) soulignant la préférence rurale de $\mathrm{E}$.

Si les distances sont relativement faibles, les points livrés sont nombreux (47 au total) et répartis sur un territoire qui couvre $10 \mathrm{EPCl}$. E met ainsi à profit une typicité alimentaire: la présence d'un type de restauration régionale bénéficiant d'un réseau dense, y compris dans les espaces ruraux. D'un point de vue logistique, toutes les livraisons de cette productrice sont effectuées en tournée. Ces dernières sont pensées les unes par rapport aux autres, afin de réduire les coûts imposés par de multiples trajets dans un tissu commercial lâche auprès d'un débouché qui se caractérise par des volumes commandés moyens, voire faibles. Par ailleurs, l'entretien a révélé que cette productrice a procédé progressivement : en consolidant d'abord ses débouchés les plus proches (et donc les moins coûteux a priori) et en ajoutant au fur et à mesure des points de plus en plus éloignés, procédant de proche en proche, puis par secteurs. Ceci lui permet de bâtir une organisation logistique en marguerite ${ }^{8}$ (REALISAB, 2013) et de livrer de nombreux points dans un territoire relativement étendu.

La stratégie de mobilité de E s'adapte aux spécificités, aux allures de contraintes, de son territoire d'appartenance, comme $F$, mais selon des orientations bien différentes. Se met en place une logique de proximité géographique à la fois subie et recherchée. La proximité géographique est privilégiée, mais impose de dessiner un territoire de mobilité étendu et dispersé. Les points livrés sont nombreux et répartis sur de multiples secteurs, mais maintenus dans des distances raisonnables. Notons, par ailleurs, que E se situe à la lisière entre deux bassins de consommation différenciés (Cambrésis et Avesnois). Elle fréquente les deux, mais avec une attraction plus forte pour le second, le plus rural des deux. On peut alors supposer qu'outre la recherche de la proximité géographique d'un bassin de consommation spécifique, s'exprime ici la fuite de la proximité géographique (subie donc) des bassins de production concurrentiels (Cambrésis et Plaine de Scarpe). La carte 6 met en évidence une certaine correspondance entre les secteurs producteurs de pommes de terre et les secteurs les moins livrés par $\mathrm{E}$. De la même façon, elle refuse de se rendre en Belgique, en raison de la concurrence perçue comme très offensive.

Carte 6. La répartition des livraisons de E au regard de la production de pommes de terre dans son territoire de mobilité.

\footnotetext{
${ }^{8}$ Il s'agit d'une organisation en secteurs de livraison. Tandis que le cœur de la marguerite représente la ferme, chaque pétale figure un secteur de livraison.
} 


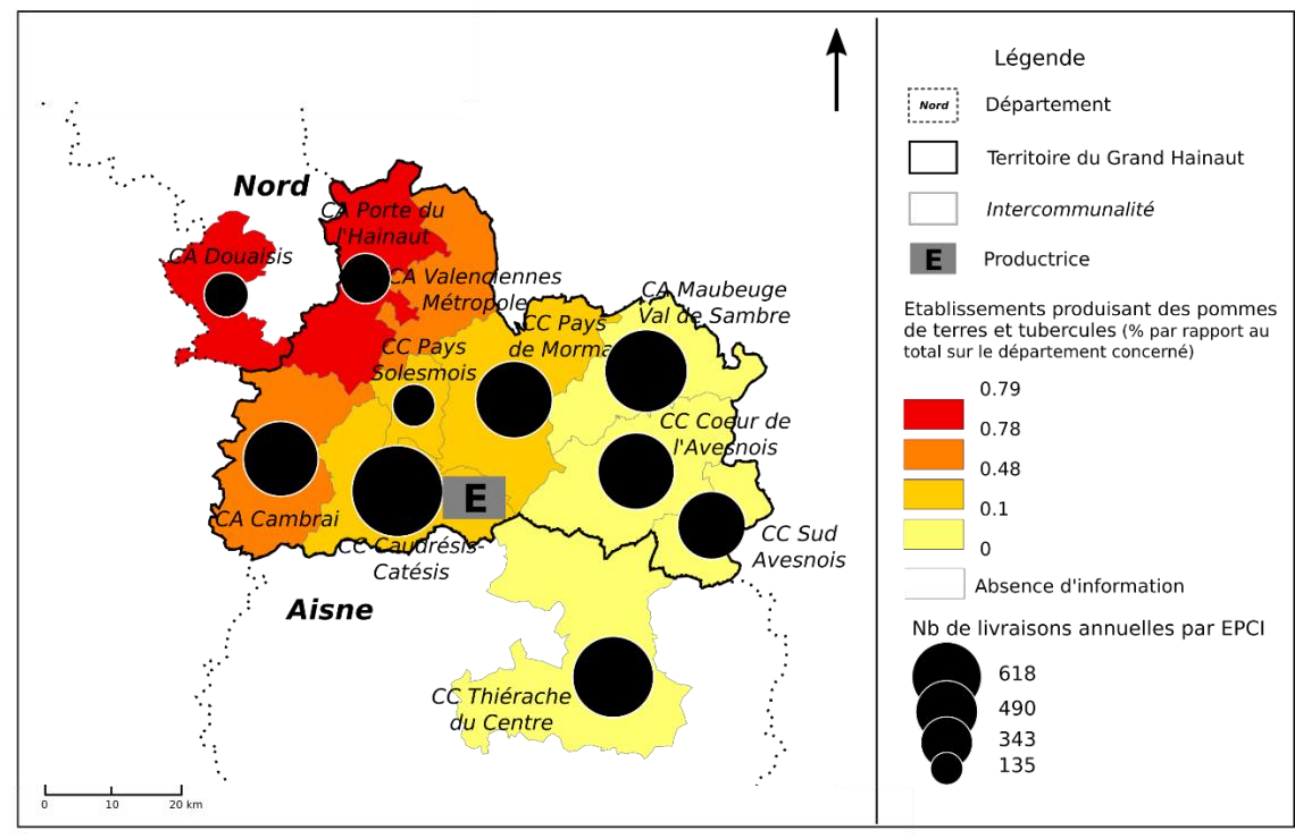

Conception : Céline Raimbert, (Philcarto). Sources : Agreste, 2010 et enquêtes COLCICCA.

\section{IV.3 Les cas de C, D : des territoires multipolarisés entre ville et campagne}

Carte 7. Les territoires de mobilité de C et D

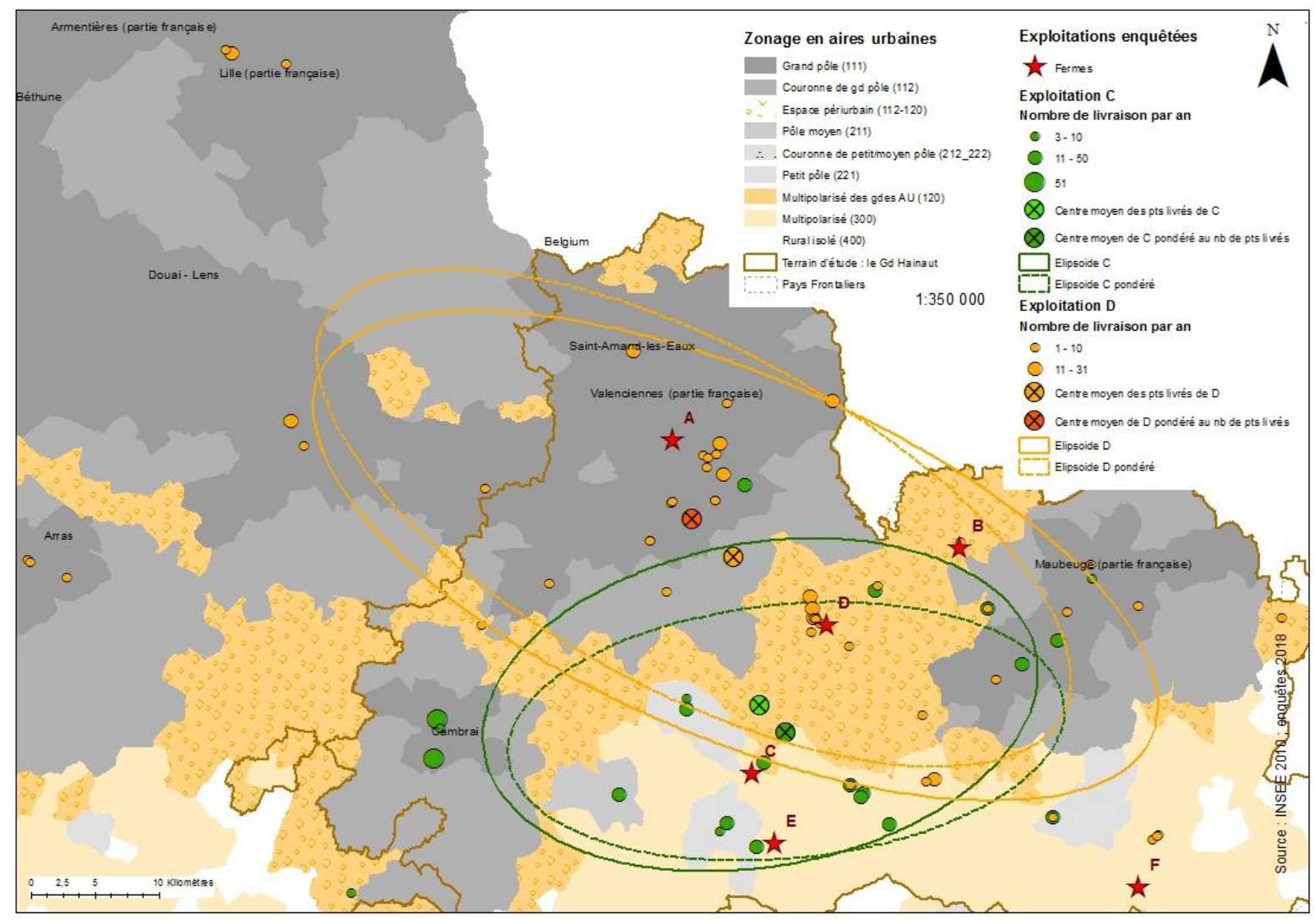

Conception : Gwenaëlle Raton IFSTTAR. Sources : INSEE, 2010 et enquêtes COLCICCA

Le cas de $C$ : un territoire orienté et un tropisme urbain affirmé quoique sans préférence de taille de pôle 
C est une jeune productrice, installée en GAEC, qui a ouvert un atelier de transformation laitière pour diversifier les activités de l'exploitation et l'ouvrir aux CCAP. Issue d'une famille très impliquée dans les organisations professionnelles locales, $\mathrm{C}$ vend, de façon privilégiée, ses produits à des commerçants détaillants fermiers. Sa ferme bénéficie d'une localisation similaire à celle de B: quoique située dans une " autre commune multipolarisée ", elle se trouve à proximité immédiate des espaces multipolarisés des grands pôles (Carte 7). Comme B donc, son territoire de mobilité est moyennement concentré. La distance médiane reste relativement faible, de même que celle entre le centre moyen et la ferme.

Pourtant, C livre un plus grand nombre d'EPCI (8) et la proportion des livraisons dans l'EPCI d'appartenance passe sous la barre des $50 \%$. Le territoire de mobilité de $C$ s'inscrit dans des espaces hétérogènes (Tableau 2) et se distingue par une polarisation multidirectionnelle, mais affirmée vers les pôles, de toutes tailles (65\% des livraisons).

Tableau 2 Caractéristiques des pôles livrés par C. Source : enquête COLCICCA

\begin{tabular}{|c|c|c|c|}
\hline Nom du pôle & Catégorie du pôle & \% des livraisons totales & Distance à la ferme \\
\hline 1. Cambrai & Grand pôle & 14,5 & 29 \\
\hline 2. Maubeuge & Grand pôle & 13,5 & 37 \\
\hline 3. Le Cateau-Cambrésis & Petit pôle & 11 & 5 \\
\hline 4.Solesmes & Petit pôle & 7 & 9 \\
\hline 5. Caudry & Moyen pôle & 6,5 & 14 \\
\hline 5. Avesnes-sur-Helpe & Petit pôle & 6,5 & 30 \\
\hline 7. Valenciennes & Grand pôle & 5 & 33 \\
\hline
\end{tabular}

Une préférence pour les grands pôles apparaît: le pôle préféré, Cambrai, est le plus proche, suivi par Maubeuge. Valenciennes fait exception. En dépit d'une distance équivalente, le pôle semble évité. Parmi les trois grands pôles livrés, Valenciennes constitue l'AU la plus peuplée (plus de 350000 habitants, contre environ 65000 pour Cambrai et 130000 pour Maubeuge). On peut supposer que la taille de Valenciennes et les contraintes logistiques que cela impose (congestion urbaine) agissent comme un repoussoir. De façon secondaire, c'est la proximité qui est privilégiée : les petits pôles, plus proches de l'exploitation (moins de 10 $\mathrm{km})$, sont livrés plus fréquemment que le moyen pôle.

Pour compléter l'analyse du territoire de mobilité de $\mathrm{C}$, nous mobilisons l'échelle des $\mathrm{EPCl}$, plus adaptée à la description des mobilités en espaces multipolarisés (Tableau 3).

Tableau 3. Principales caractéristiques des trois sous-territoires de mobilité de C

\begin{tabular}{|c|c|c|c|}
\hline Territoire & EPCl correspondants & $\begin{array}{c}\% \text { du total } \\
\text { des } \\
\text { livraisons }\end{array}$ & $\begin{array}{c}\text { Distance } \\
\text { moyenne }\end{array}$ \\
\hline (1) & CC Pays de Mormal & $34 \%$ & $17 \mathrm{~km}$ \\
\hline (2) & CA Cambrai / CC Caudrésis-Catésis & $32 \%$ & $21 \mathrm{~km}$ \\
\hline (3) & $\begin{array}{c}\text { CA Maubeuge Val de Sambre / CC Cœur de } \\
\text { I'Avesnois / CC Pays Solesmois / CA Valenciennes } \\
\text { Métropole / CA Porte du Hainaut }\end{array}$ & $14 \%$ & $23 \mathrm{~km}$ \\
\hline
\end{tabular}

\section{Source : Enquête COLCICCA}

Une tripartition du territoire apparaît, les livraisons se destinent à :

(1) De façon préférentielle, au territoire d'appartenance. En son sein, les livraisons dans les « autres communes polarisées ", catégorie à laquelle appartient la commune de la ferme de C, sont majoritaires (58\% des livraisons au sein de l'EPCI). Ce territoire répond à une logique de proximité géographique immédiate. La productrice s'appuie, en premier lieu, sur le bassin de consommation auquel elle appartient. Cela semble le résultat de la mobilisation d'un réseau social professionnel dense. 
Au-delà de ce territoire de prédilection, C mène une stratégie de diversification et fréquente d'autres territoires :

(2) Un territoire proche et orienté vers le Cambrésis et, en cela, choisi. D'un point de vue du bassin de consommation, il concentre plusieurs pôles de diverses tailles (Cambrai, Caudry et Le Cateau-Cambrésis) qui connaissent une renaissance économique (INSEE, 2015b). D'un point de vue du bassin de production, le Cambrésis, davantage tourné vers la polyculture, permet à $C$ de s'ouvrir à un marché perçu comme moins saturé que l'Avesnois. D'un point de vue logistique, C livre majoritairement en tournée (82,5\%), les traces directes correspondant à des cas spécifiques (lieux très proches et fréquentés pour d'autres activités).

(3) Des territoires de diversification qui se composent de points éparpillés sur un espace relativement large, sans orientation claire si ce n'est que les livraisons se concentrent exclusivement dans des pôles.

\section{IV.3.2 Le cas de D : un territoire de tropisme urbain multipolarisé mais concentré sur les grands pôles}

D est une exploitation de lycée agricole. Outre des objectifs de rentabilité économique, $D$ remplit une mission de développement local du territoire, propre à influencer le choix des lieux fréquentés. En termes de localisation, elle se situe dans un espace similaire à B, soit un espace multipolarisé (Carte 7). Comme B (et dans une moindre mesure $C$ ), deux bassins de consommation lui sont accessibles: Maubeuge et Valenciennes. Pourtant, $D$ ne semble pas adopter la même stratégie de concentration que $B$. Le nombre de points livrés est bien plus élevé (50), de même que le nombre d'EPCI (10). D semble ainsi davantage se rapprocher de $C$, pour son attrait pour des pôles multiples. D livre, de façon privilégiée, les grandes AU (89\% des livraisons), majoritairement leurs grands pôles (56\%), mais aussi leur couronne ou des espaces multipolarisés des grandes AU (33\%). Avec des livraisons réparties dans 6 grands pôles différents, le territoire de mobilité de $D$, tout comme celui de $\mathrm{C}$, fait montre d'une hétérogénéité spatiale certaine, qui s'exprime aussi à travers la tripartition de son territoire de mobilité :

(1) Une première polarité autour du territoire d'appartenance : 34\% des livraisons effectuées dans l'EPCI d'appartenance. La très grande majorité des livraisons $(73,5 \%)$ a pour destination la commune de l'exploitation, une "commune multipolarisée des grandes $A U$ ", qui bénéficie d'une clientèle potentielle relativement importante.

(2) Une préférence secondaire pour la partie nord du département, sa portion la plus urbanisée. Les livraisons se répartissent sur 5 des 6 grands pôles livrés (Tableau 4).

Tableau 4. Caractéristiques des pôles livrés par D dans son sous-territoire de prédilection. Sources : enquête COLCICCA \& INSEE, 2015.

\begin{tabular}{|c|c|c|c|}
\hline Grands pôles & $\begin{array}{c}\text { \% des livraisons à } \\
\text { destination du secteur } \\
\text { nord }\end{array}$ & Distance à l'exploitation & $\begin{array}{c}\text { Population } \\
\text { (nb hab.) }\end{array}$ \\
\hline 1. Valenciennes & $56 \%$ & $20 \mathrm{~km}$ & 369849 \\
\hline 2. Douai-Lens & $16 \%$ & $57 \mathrm{~km}$ & 539715 \\
\hline 3. Lille & $13 \%$ & $69 \mathrm{~km}$ & 1184708 \\
\hline 4. Arras & $8 \%$ & $97 \mathrm{~km}$ & 130541 \\
\hline 5. Saint-Amand-les-Eaux & $7 \%$ & $33 \mathrm{~km}$ & 33873 \\
\hline
\end{tabular}

À l'exception d'un cas, plus l'AU est proche de la ferme de D, plus la fréquence des livraisons est élevée.

(3) Les livraisons restantes (20\%) se font en majorité vers des espaces hors aires d'influence (55\%) compris dans le territoire du Grand Hainaut, les autres sont réparties entre les AU de Maubeuge (22\%) et d'Avesnes-surHelpe (1\%) et des espaces périurbains (22\%). Dans ce cas, aucune stratégie ne semble clairement émerger, et ce d'autant moins que ces points sont en fait livrés occasionnellement. Le Grand Hainaut apparaît comme un espace relativement délaissé par $D$, malgré sa proximité avec l'exploitation. On en déduit ainsi une proximité géographique qui semble donc subie : D préfère parcourir de plus grandes distances et se tourner vers le nord du département et l'ensemble des grands pôles les plus proches (moins de 100 km). 


\section{Discussion}

L'originalité de notre approche tient à l'observation d'un phénomène qui rompt avec les logiques connues de l'approvisionnement alimentaire (le sens de la relation) et nécessite une approche par les mobilités et par l'espace produit par ces mobilités. Plusieurs éléments méritent ainsi d'être discutés: d'un point de vue thématique, pourquoi les acteurs de la production sont-ils aujourd'hui des acteurs de la distribution et qu'induit ce changement de pratiques ? ; d'un point de vue méthodologique, en quoi et dans quelle mesure les mobilités des agriculteurs permettent-elles d'appréhender les territoires alimentaires et leurs enjeux spécifiques?

\section{V.I Réflexion autour des déterminants des mobilités des producteurs agricoles en CCAP}

La réduction du nombre d'intermédiaires a produit une révolution silencieuse sur les exploitations agricoles en CCAP : ne pouvant pas toujours écouler l'ensemble de la production sur la ferme et devant fournir des produits directement consommables et transportables, les producteurs ajoutent un grand nombre de tâches à leur métier. Dans ce contexte, le rôle dévolu aux producteurs dans la constitution de territoires de l'alimentation de proximité devient central. Ils sont ainsi amenés à (re)questionner les potentialités du territoire : à qui commercialiser? A quel prix ? Où sont localisés les consommateurs cibles ? Mais aussi : qui sont mes concurrents, où sont-ils concentrés ? Pour un producteur, les espaces potentiels de distribution des produits en CCAP sont constitués du tissu commercial composé de points de vente proposant des produits locaux, plus ou moins accessibles selon la localisation de la ferme.

La localisation des points qu'il est possible de livrer répond ainsi aux effets d'attractivité déjà théorisés en géographie. On peut se référer à ceux des lieux centraux sur une périphérie, résultats de la concentration de populations cibles et de moyens disponibles pour y accéder. Dans le cadre de flux initiés depuis des milieux ruraux ou périurbains, on peut définir cette attractivité comme un tropisme urbain. Le phénomène est présent chez la plupart des productrices enquêtées, quoique de façon exacerbée dans les cas $A$ et $B$, pour lesquels le coût d'opportunité d'accès à un tissu commercial dense est a priori moindre en raison de leur proximité à cette dernière. Un autre modèle géographique intéressant pour nos cas serait celui de Christaller qui insiste sur la hiérarchie des réseaux urbains. Alors que les petites villes concentrent les biens les plus demandés et les plus concurrencés, les plus grandes concentrent les biens les plus rares et valorisés. Or, on a pu remarquer que les productrices réalisaient des arbitrages entre pôles urbains de tailles diverses. Néanmoins, si les théories géographiques sont éclairantes, elles n'expliquent pas toutes les stratégies. Notre travail montre que d'autres variables interviennent.

\section{La localisation de la ferme}

Les stratégies mises en évidence dans l'étude de cas montrent une adaptation des productrices aux opportunités et contraintes qu'offre la situation de la ferme. Les productrices $A$ et $B$, bénéficiant d'une position centrale, suivent des stratégies de concentration en milieu urbain et dessinent des territoires de mobilité peu étendus, homogènes et dont le point central est la ferme elle-même. Les productrices $E$ et $F$ se situent, pour leur part, dans des espaces marginaux. Elles adoptent des stratégies très différentes mais marquées et unidirectionnelles, consistant, pour $E$, en une stratégie de concentration en milieu rural et, pour $F$, en une stratégie d'extraversion. Dans les deux cas, les territoires de mobilité sont plutôt étendus et relativement homogènes. Enfin, la situation duelle des fermes de $C$ et $D$, entre ville et campagne, met en évidence des profils moins marqués suivant des stratégies de diversification. Outre une préférence pour le local et les pôles, les productrices oscillent entre concentration pour $C$ et extraversion pour $D$, cette stratégie secondaire influant sur la forme de leur territoire de mobilité, comparables respectivement à ceux de $E$ et $F$ (carte 8). Au-delà de ces cas, c'est le lien entre la localisation de la ferme et le type de stratégie spatiale qui est mis en évidence. II évoque l'existence d'un gradient ville/campagne: les fermes situées dans l'aire d'influence d'un grand pôle peuvent mener des stratégies de relocalisation de leurs débouchés, alors que les fermes localisées en marge y parviendraient plus difficilement et seraient conduites à mener des stratégies duelles de relocalisation, mais aussi de consolidation via des pratiques d'extraversion par exemple. 


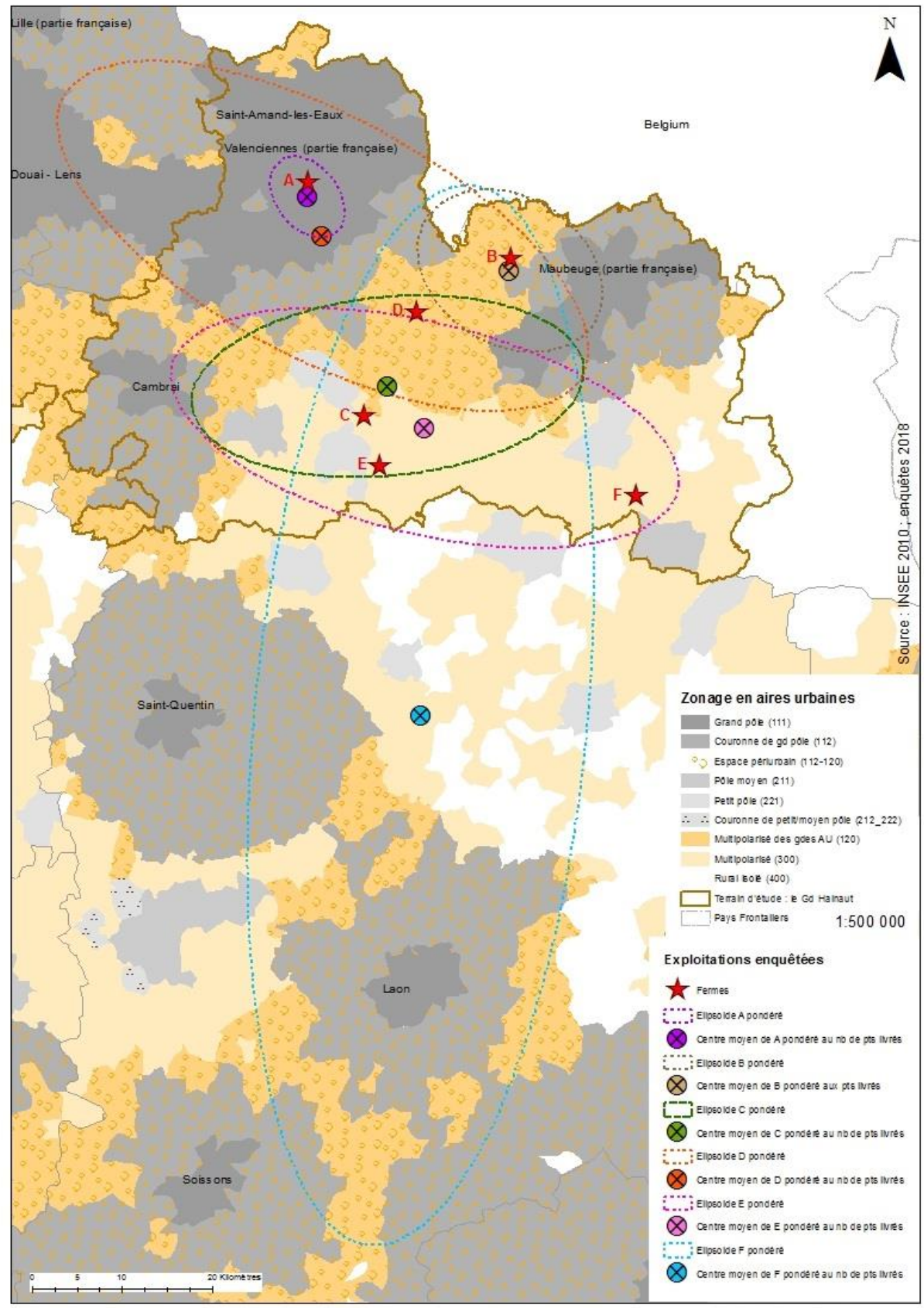

Conception : Gwenaëlle Raton IFSTTAR. Sources : INSEE, 2010 et enquêtes COLCICCA

\section{Les bassins de production}

Outre les effets d'attractivité à l'échelle des bassins de consommation, l'étude de cas montre des effets de concurrence, à l'échelle des bassins de productions, qui peuvent également traduire une survalorisation de la connaissance de leur territoire d'appartenance d'un point de vue agricole (comparativement à la connaissance des bassins de consommation). La plupart des productrices enquêtées (C, D, E et F) exprime une préférence à l'égard de territoires dont l'orientation productive diffère de la leur. Elles privilégient des espaces où elles considèrent non seulement que leurs produits pourront bénéficier d'un effet de rareté plus grand, mais aussi 
que leurs concurrents directs seront moins nombreux. Ainsi, C et D, productrices de produits laitiers orientent leurs livraisons en-dehors de l'Avesnois, terre d'élevage. Pour la productrice de yaourts, les pôles divers de tailles modestes sont privilégiés; pour la productrice de fromages, bénéficiant d'un effet de rareté plus grand, les distances parcourues sont plus grandes et les pôles fréquentés plus grands (elle est la seule productrice à livrer jusqu'à Lille).

Via la prise en compte de la localisation des fermes et des bassins de production, les études de cas montrent l'importance de la distance dans les choix. Trois façons de la pondérer interviennent alors, en prenant en compte :

\section{$\underline{\text { La contrainte des coûts logistiques }}$}

Les coûts logistiques concernent aussi bien les dépenses financières engagées que le temps consacré par les producteurs aux tâches logistiques. C'est un ensemble de variables qu'il faut considérer comme le type de produits et les quantités livrées (transformés ou non, nécessitant un transport frigorifique ou du transport amont, durée de la DLC, saisonnalité), l'organisation de la distribution (véhicule utilisé, trace directe, tournée, taux de remplissage, retours à vide) et l'organisation de la commercialisation (vente en paniers allongeant les temps de préparation, caractéristiques du lieu livré et notamment, conditions de circulation, service de gestion des flux). Les contraintes logistiques vont ainsi avoir un impact sur les choix réalisés par les producteurs : choisir de livrer en ville, parfois beaucoup plus loin pour multiplier les débouchés (cas de F) ou au contraire l'éviter pour ne pas perdre du temps dans le trafic (cas de C), préférer des villes moyennes aux plus grandes, choisir de développer un territoire pour créer des secteurs de livraison et privilégier les tournées (cas de $C$ et $E$ ). La perception de la distance est ainsi aussi liée à la perception des contraintes logistiques dans différents contextes. De ce fait, la maturité de l'organisation logistique et commerciale influence fortement la taille du territoire de mobilité, celui-ci étant le résultat de réajustements constants, créant des territoires évolutifs (extension ou resserrement).

\section{$\underline{\text { Les représentations territoriales et les effets de proximité }}$}

C'est là qu'intervient la dimension idéelle du territoire. Un lieu peut paraître lointain parce qu'il n'est pas familier et que des projections sur le type de clientèle $s^{\prime} y$ trouvant s'avèrent difficiles. On retrouve dans les études de cas une tendance à la fréquentation des milieux similaires au sien (cas de $A, B$, E et dans une moindre mesure $C$ et D).

Dans les cas d'étude, la distance perçue semble compter au moins autant que la distance réelle (les lieux proches le sont d'autant plus qu'ils ressemblent au territoire d'appartenance) et la proximité géographique est recherchée, dans le sens où elle est mise en valeur et exploitée par les productrices qui la considèrent comme un atout. Lorsqu'il s'agit de proximités urbaines, la logique apparaît très forte (cas de $A$ et $B$ ), dans la mesure où la ville concentre a priori un potentiel de bénéfices importants. Mais la proximité urbaine peut aussi apparaître subie, comme le démontre le cas de $C$ qui évite Valenciennes : la ville n'est pas si lointaine, mais est perçue comme peu ou pas accessible. Lorsqu'il s'agit de proximités rurales, ces dernières peuvent apparaître recherchées, $c^{\prime}$ est le cas de $E$, qui y voit des caractéristiques spécifiques attractives en termes d'accessibilité (moindres distances) et de débouchés-cibles (densité des friteries) (Goffette-Nagot, Schmitt, 2005). A l'inverse, $\mathrm{F}$ considère ces proximités comme subies et elle privilégie une logique d'extraversion. Pour autant, ces stratégies d'extension du territoire de mobilité n'excluent pas de fait une concentration parallèle dans le territoire d'appartenance. Le jeu des proximités urbaines/rurales, recherchées/subies permet ainsi de caractériser les stratégies des producteurs.

Ces trois façons de pondérer la distance contribuent, à des degrés divers, à défaire les logiques d'attractivité classiques observées dans les modèles géographiques.

\section{V.2. Originalité et enjeux de l'approche par les mobilités}

Sur le plan méthodologique, l'originalité de notre approche tient à la possibilité de croiser territoire de mobilité des agriculteurs et territoires alimentaires locaux en construction. Les études de cas montrent, qu'au-delà de pratiques individuelles, fruits des dimensions à la fois matérielles et idéelles des territoires, des processus communs sont identifiables et que ces mobilités constituent des facteurs de reconfigurations sociales et territoriales (Caron, Torre, 2005). Néanmoins, les systèmes alimentaires locaux ne peuvent s'appréhender par les seules mobilités individuelles de producteurs (Brives et al., 2017). En outre, le nombre de cas étudiés est limité, en nombre, en diversité de profils, en diversité de territoires (un terrain d'étude multipolarisé). Pour contourner ces limites deux démarches peuvent être envisagées : augmenter le nombre de trajets étudiés ; 
enrichir cette approche de la mobilité d'individus en la combinant avec une approche réseau. II s'agirait alors de voir la façon dont les réseaux supportent les mobilités en agrégeant à la connaissance des mobilités, la qualification des nœuds et des relations, notamment dans le cadre de collectifs mutualisant.

\section{Conclusion : mobilités des agriculteurs et construction de territoires alimentaires locaux, quels enjeux ?}

Les cas d'étude illustrent des adaptations des productrices aux opportunités et contraintes qu'offre la localisation de la ferme. Au-delà des pratiques observées, l'analyse des territoires de mobilité construits mais aussi des lieux non livrés informe sur les enjeux actuels des systèmes alimentaires locaux émergents. Ainsi, aux côtés des questions mises en évidence dans la littérature comme celle des inégalités économiques et sociales auxquelles sont confrontées producteurs et consommateurs des CCAP et celle de l'impact d'un changement d'échelle des CCAP sur les inégalités préexistantes (Deverre, Lamine, 2010 ; Chiffoleau 2012), notre étude pointe spécifiquement deux types d'inégalités d'ordre spatial :

- des inégalités spatiales touchant directement les producteurs selon la localisation de leur ferme et impactant leur organisation et donc la rentabilité de leurs activités ;

La première tient au constat d'inégalités d'accès aux bassins de consommateurs en fonction de la localisation de la ferme ; le poids des distances parcourues et des tâches logistiques n'impactant pas tous les producteurs de la même manière. Au-delà des différenciations productives, les différenciations spatiales à l'œuvre dans la construction des systèmes alimentaires locaux sont donc à prendre en compte. Derrière ce constat semble ainsi se jouer toute la question des distances réelles et idéelles entre certaines fermes et certains bassins de consommation et des barrières de connaissance réciproques pouvant expliquer certaines difficultés de rencontre entre offre et demande de produits locaux.

- $\quad$ et des inégalités spatiales en termes d'offres diversifiées de produits locaux sur des territoires non privilégiés par les producteurs.

La seconde tient au constat que les producteurs, eux-mêmes objets d'inégalités spatiales, évoquent des pratiques de contournements de certains lieux/clients pour des raisons de pauvreté perçue, de contexte socioéconomique non favorable à la rencontre effective d'une clientèle. Ainsi, une autre réalité des CCAP aujourd'hui serait l'existence de pratiques d'évitements, d'exclusions, qui, au final, sont à même de produire, de créer ou d'amplifier des inégalités spatiales et territoriales. Ce phénomène renvoie plus généralement à l'inégalité d'accès à l'alimentation de qualité, pouvant entraîner des injustices alimentaires. Faute d'action politique en la matière, ce sont tour à tour la fragilité des exploitations en CCAP et les inégalités d'accès aux produits locaux qui pourraient caractériser ces chaînes dans une optique de changement d'échelle. Le poids des représentations risque en outre de prédominer, rendant ces circuits plus élitistes.

Une autre piste à investir pourrait être la question de la force des relations villes/campagne. Dans quelle mesure les fermes les plus distantes (dimension réelle et idéelle de la distance ici) des bassins de consommation ne sont-elles pas également celles avec qui la force des relations avec les consommateurs urbains en général est moindre? Dans quelle mesure certaines exploitations pâtissent d'un manque d'antériorité de relations nouées avec le tissu urbain et ce, quels que soient les motifs ?

Le point de vue logistique, valorisé dans cet article, donne des arguments supplémentaires pour pointer le besoin de renforcement des relations ville/campagne. Les travaux sur les contraintes logistiques illustrent déjà des déséquilibres: les retours à vides nombreux semblant marquer une absence de réciprocité dans les échanges ruraux/urbains. Le constat du poids de la dimension idéelle sur les décisions de mobilité des agriculteurs, doublé de celui d'une certaine inclinaison à livrer les espaces similaires au lieu de vie ou aux lieux connus semble montrer l'urgence à recréer des liens forts.

Dans un contexte de valorisation du manger local, ce constat tend à souligner le rôle à jouer des collectivités territoriales pour rendre attractifs les espaces les plus divers et ainsi garantir aux producteurs des débouchés proches et rentables et aux consommateurs des produits locaux diversifiés et accessibles. Cela peut passer par des aides apportées à la constitution de collectifs de producteurs, à même de répartir les coûts logistiques, voire de collectifs mixtes (producteurs/consommateurs) tels les épiceries participatives. Elle peut aussi s'appuyer, dans l'esprit de la loi EGALim, sur des incitations auprès des restaurations collectives, à tous les échelons territoriaux, à privilégier l'approvisionnement local. 


\section{Bibliographie}

AGRESTE, 2012, Nord-Pas-de-Calais. Données, n6, 6 p.

AGRESTE, 2014, Picardie. Mémento de la statistique agricole, Amiens, DRAAF.

ALLIX A., 1923, Les foires. Etude géographique. La géographie, 43 pp. 521-563.

AGUILERA A., F. De CONINK, P. HAUCHARD, 2007, Le rôle des déplacements professionnels dans les entreprises industrielles multi-établissements. Le cas d'un fournisseur de l'automobile, Recherche-Transport-Sécurité, 14(96), p. 195-209.

BAHOKEN F., G. RATON, GRESSEL R., 2018, Morphologie des mobilités spatiales de professionnels mobiles. Vers la représentation de "structures territoriales d'interventions professionnelles, CIST 2018, Représenter les territoires, 22 \& 23 mars 2018, Université de Rouen Normandie, 6 p.

BAYSSE-LAINE A., 2017, L'empreinte spatiale des approvisionnements alimentaires locaux: un modèle graphique, M@ppemonde, $\mathrm{n}^{\circ} 122$.

BAYSSE-LAINE A., C. PERRIN,2017, Les espaces agricoles des circuits de proximité : une lecture critique de la relocalisation de l'approvisionnement alimentaire de Millau, Natures Sciences Sociétés, Vol. 25, p.21-35.

BELTON L. et F. De CONINK, 2007, Des frontières et des liens, Les topologies du privé et du professionnel pour les travailleurs mobiles, Réseaux, $n^{\circ} 140$, p. 67-100.

BLANQUART C, A. GONCALVES, G. RATON, L. VAILLANT, 2015, Vecteurs et freins d'une logistique plus durable dans les circuits-courts : le cas du Nord-Pas-de-Calais, $52^{e}$ colloque ARSDLF, 7-9 juillet 2015, Montpellier.

BOGNON S., 2015, Nourrir Paris : trajectoire de l'approvisionnement alimentaire de la métropole capitale, de la fin de l'Ancien Régime à nos jours, Géocarrefour, 90/2, p. 163-171.

BOGNON S., 2014. Les transformations de l'approvisionnement alimentaire de la métropole parisienne. Trajectoire socioécologique et construction de proximités. Thèse de doctorat, Paris, Université Paris 1 Panthéon-Sorbonne.

BOGNON S., S. BARLES, G. BILLEN, J. GARNIER, 2017, Approvisionnement alimentaire parisien du XVIIle au XXIe siècle : les flux et leur gouvernance. Récit d'une trajectoire socioécologique ?, Natures Sciences Sociétés, Vol. 26, p. 17-32.

BRIVES H., C. CHAZOULE, P. FLEURY, P. VANDENBROUCKE, 2017, La notion d' « agriculture du milieu » est-elle opérante pour l'analyse de l'agriculture de Rhône-Alpes ?, Economie rurale, 357-358, p. 41-56.

CARON A., A. TORRE, 2005, Conflits d'usage et de voisinage dans les espaces ruraux. In FILIPPI M. et al. Proximités et changements socio-économiques dans les mondes ruraux, Quae, p. 297-314.

CHALEARD J.-L., 1996, Temps des villes, temps des vivres, l'essor du vivrier marchand en Côte d'lvoire, Paris, Karthala, $661 \mathrm{p}$.

CHIFFOLEAU, 2012, Circuits courts alimentaires, dynamiques relationnelles et lutte contre l'exclusion en agriculture, Economie rurale, $\mathrm{n}^{\circ} 332$, p. 88-101.

COFFI DIT GLEIZE S., 2012, Étude des circuits de commercialisation de la filière fruits de la région Centre, Mémoire de fin d'études, Agrocampus Ouest, $69 \mathrm{p}$.

DELMAS C., 2015, "Une profession tournée vers l'avenir : sociologie des mutations notariales », La Semaine juridique notariale et immobilière, $\mathrm{n}^{\circ} 36$.

DEVERRE, C. LAMINE C., 2010, Les systèmes agroalimentaires alternatifs. Une revue de travaux anglophones en sciences sociales, Economie rurale, 317, p. 57-73.

DI MEO G., P. BULEON, 2005, L'espace social. Lecture géographique des sociétés, Paris, Armand Colin, 304 p.

FILIPPINI R., E. MARRACCINI, M. HOUDART, E. BONARI \& S. LARDON, 2016, Food production for the city: hybridization of farmers' strategies between alternative and conventional food chains, Agroecology and Sustainable Food Systems, 40:10, p. 1058-1084. 
GONCALVES A., 2013, La performance des organisations logistiques des circuits courts de distribution : une analyse des déterminants et leviers d'amélioration, Thèse de doctorat, Université de Lille 1, 459 p.

GOFFETTE-NAGOT F. et SCHMITT B., 2005, Les proximités, la ville et le rural. In FILIPPI M. et al. Proximités et changements socio-économiques dans les mondes ruraux, Quae, p. 193-199.

GRESSEL R., MUNDETEGUY C., 2008, Les professionnels mobiles, Un groupe hétérogène avec une exposition importante au risque routier, Recherche transports sécurité, $n^{\circ}$ 99, p. 147-167.

HERVIEU B., 2002, La multifonctionnalité de l'agriculture : genèse et fondements d'une nouvelle approche conceptuelle de l'activité agricole, Cahiers Agricultures, n¹1, p. 415-419.

INSEE, 2015a, SCoT Sambre-Avesnois : de la fragilité sociale à l'éloignement social. In. INSEE, Dossier Nord-Pasde-Calais, p. 80-87.

INSEE, 2015b, SCoT du Cambrésis : un essor résidentiel autour de Cambrai, les zones rurales en situation d'isolement In. INSEE, Dossier Nord-Pas-de-Calais, p. 40-47.

INSEE, 2015c, Le zonage en aires urbaines. In INSEE, INSEE Méthodes, Les zonages d'étude de l'Insee. Une histoire des zonages supra communaux définis à des fins statistiques, $\mathrm{n}^{\circ} 129, \mathrm{p} .11-40$.

LAMINE C. et CHIFFOLEAU Y., 2012, Reconnecter agriculture et alimentation dans les territoires : dynamiques et défis, POUR, Vol. 3, n²15-216, p. 85-92.

LE GALL. J., 2011, Buenos Aires maraîchère : une Buenos Aires bolivienne ?: le complexe maraîcher de la région métropolitaine à l'épreuve de nouveaux acteurs, Thèse de doctorat, Université Panthéon-Sorbonne -Paris 1; Universidad de Buenos Aires, 776 p.

LEVY J., M. LUSSAULT, 2003, Dictionnaire de la géographie et de l'espace des sociétés, Paris, Belin, 1228 p.

LOMBARD J., NINOT O., 2010, Mobiles des Suds, mobiles au Sud. Editorial, Espace Populations Sociétés,

no2-3, p. 155-165.

NINOT O., 2003, Vie de relations, organisation de l'espace et développement en Afrique de l'Ouest : la région de Tambacounda au Sénégal, Thèse de doctorat, Université de Rouen, IRD, $467 \mathrm{p}$.

PECQUEUR B., ZIMMERMANN J.-B. (éds.), 2004, Economie de proximités, Paris, Hermès-Lavoisier, 264 p.

PERRIER-CORNET P., 2017, Les sciences sociales et les mutations des mondes ruraux, Études rurales, $\mathrm{n}^{\circ} 200, \mathrm{p}$. 28-41.

RALLET A., TORRE A., 2004, Proximité et localisation, Economie rurale, n²28, p. 25-41.

RATON G., 2012, Les foires au Mali, de l'approvisionnement urbain à l'organisation de l'espace rural. Le cas de la périphérie de Bamako, Thèse de doctorat en géographie, Université Paris 1, 413 p.

RATON G., VAILLANT L., M'BALLA J., BOUCHEZ P., LESNARD S., ANXIONNAZ J., RIOS M., 2018, Organisations Logistiques Intelligentes des Circuits courts en vallée de Seine, rapport final OLICO-Seine, IFSTTAR-ADEME.

RATON G., GONCVALVES, A., VAILLANT L., BLANQUART, C., LOEUILLEUX P., TELLIER C., 2015). ALLOCIRCO, Alternatives logistiques pour les circuits courts en Nord-Pas de Calais, rapport final ALLOCIRCO, IFSTTAR-Région Hauts-de-France, $275 \mathrm{p}$.

REALISAB, 2013, Organiser une logistique collective de proximité, Guide et fiches pratiques, Chambre Régionale d'Agriculture de Franche-Comté, $28 \mathrm{p}$.

SCHLICH E., BIEGLER I., HARDTERT B., LUZ M., SCHODER S., SCHROEBER J. et WINNEBECK S., 2006, La consommation d'énergie finale de différents produits alimentaires : un essai de comparaison, Courrier de l'environnement de I'INRA, n53, p. 111-120

SCOT SAMBRE-AVESNOIS, 2017, Diagnostic agricole, 80 p.

TALBOT D., KIRAT T., 2005, Proximité et institutions : nouveaux éclairages. Présentation du numéro. Economie \& Institutions, $n^{\circ} 6-7$, p. 9-15.

TORRE A., 2009, Retour sur la notion de proximité géographique, Géographie, économie, société, Vol. 11, n¹, p. 63-75. 
TORRE A. et FILIPPI M., 2005, Les mutations à l'œuvre dans les mondes ruraux et leurs impacts sur l'organisation de l'espace. In FILIPPI M. et al. Proximités et changements socio-économiques dans les mondes ruraux, Quae, p. 1-36.

\section{Annexes}

Tableau A. Caractéristiques des points livrés par productrice. Source : enquêtes COLCICCA

\begin{tabular}{|c|c|c|c|c|c|c|c|c|}
\hline Productrice & A & B & C & D & E & F & Médiane \\
\hline Nb total de livraisons par an & 709 & 307 & 728 & 326 & 3725 & 412 & $\mathbf{4 1 2}$ \\
\hline Nb total de points livrés & 12 & 18 & 26 & 50 & 47 & 19 & $\mathbf{2 2 , 5}$ \\
\hline \multirow{2}{*}{$\begin{array}{c}\text { Distance exploitation- } \\
\text { point livré (km) }\end{array}$} & Distance médiane & 5 & 7 & 12 & 17 & 21 & 65 & $\mathbf{1 4 , 5}$ \\
\cline { 2 - 10 } & Distance maximale & 20 & 51 & 45 & 91 & 46 & 107 & $\mathbf{4 8 , 5}$ \\
\hline \multirow{2}{*}{$\begin{array}{c}\text { Localisation des points } \\
\text { livrés }\end{array}$} & Nb total EPCI livrés & 2 & $6(+1)$ & 8 & 10 & 10 & 10 & $\mathbf{8}$ \\
\cline { 2 - 10 } & $\begin{array}{c}\text { \% de livraisons dans } \\
\text { l'EPCl d'appartenance }\end{array}$ & 84 & 64 & 34 & 39 & 16,5 & 12 & $\mathbf{3 6 , 5}$ \\
\hline $\begin{array}{c}\text { Type d'organisation } \\
\text { logistique (\% du total } \\
\text { des livraisons) }\end{array}$ & En tournée & 40 & 45 & 82,5 & 87 & 100 & 61 & $\mathbf{7 2}$ \\
\cline { 2 - 10 } & En trace directe & 60 & 55 & 17,5 & 13 & 0 & 39 & $\mathbf{2 8}$ \\
\hline
\end{tabular}

Tableau B. Les points livrés des productrices au regard du zonage en AU.. Source : enquêtes COLCICCA.

\begin{tabular}{|c|c|c|c|c|c|c|c|c|}
\hline \multicolumn{2}{|c|}{ Productrice } & A & B & $\mathrm{C}$ & D & $\mathbf{E}$ & $\mathbf{F}$ & Médiane \\
\hline \multicolumn{2}{|c|}{ AU d'appartenance } & $\begin{array}{l}\text { Grand pôle } \\
\text { urbain (111) }\end{array}$ & $\begin{array}{c}\text { Commune } \\
\text { multipolarisée } \\
\text { des grands } \\
\text { pôles (120) } \\
\end{array}$ & $\begin{array}{c}\text { Autre } \\
\text { commune } \\
\text { multipolarisée } \\
(300) \\
\end{array}$ & $\begin{array}{c}\text { Commune } \\
\text { multipolarisée } \\
\text { des grands } \\
\text { pôles (120) } \\
\end{array}$ & $\begin{array}{c}\text { Autre } \\
\text { commune } \\
\text { multipolarisée } \\
(300) \\
\end{array}$ & $\begin{array}{c}\text { Autre } \\
\text { commune } \\
\text { multipolarisée } \\
(300)\end{array}$ & - \\
\hline \multirow{4}{*}{$\begin{array}{c}\text { Types d'AU } \\
\text { de } \\
\text { destination } \\
\text { (\% des } \\
\text { livraisons } \\
\text { totales) }\end{array}$} & $\begin{array}{c}\text { Grands pôles } \\
\text { urbains } \\
\text { (111) }\end{array}$ & 100 & 34 & 33 & 56 & 19 & 70 & 45 \\
\hline & $\begin{array}{c}\text { Espaces } \\
\text { périurbains } \\
(112-120)\end{array}$ & 0 & 64 & 15 & 33 & 21 & 24 & 22,5 \\
\hline & $\begin{array}{c}\text { Autres aires } \\
(211-212-221-222)\end{array}$ & 0 & 2 & 32 & 0 & 21 & 6 & 4 \\
\hline & $\begin{array}{c}\text { Espaces hors des } \\
\text { aires d'influence } \\
(300-400)\end{array}$ & 0 & 0 & 20 & 11 & 39 & 0 & 5,5 \\
\hline \multicolumn{2}{|c|}{$\begin{array}{c}\% \text { de livraisons vers } A U>100000 \\
\text { habitants }\end{array}$} & 93 & 31 & 23 & 55 & 23 & 32,5 & 31,75 \\
\hline
\end{tabular}

N.B. : Concernant la productrice B, l'un de ses points n'a pas pu être pris en compte ici car il est situé en Belgique.

Tableau C Distances entre les fermes et leurs centres moyens et pondérés.. Source : enquêtes COLCICCA.

\begin{tabular}{|c|c|c|}
\hline Productrice & $\begin{array}{c}\text { Distance ferme / } \\
\text { centre moyen }(\mathbf{k m})\end{array}$ & $\begin{array}{c}\text { Distance ferme / centre moyen } \\
\text { pondéré à la fréquence de } \\
\text { livraison }(\mathbf{k m})\end{array}$ \\
\hline A & 2,286 & 1,821 \\
\hline B & 4,561 & 1,515 \\
\hline C & 5,332 & 4,117 \\
\hline D & 9,012 & 13,362 \\
\hline E & 8,334 & 6,394 \\
\hline F & 24,941 & 34,162 \\
\hline
\end{tabular}

Tableau D Types de débouchés livrés par productrice. Source : enquêtes COLCICCA. 


\begin{tabular}{|c|c|c|c|c|c|c|c|}
\hline \multirow{3}{*}{ Productrice } & \multicolumn{7}{|c|}{ Types de débouchés livrés (\% du total des livraisons) } \\
\hline & \multicolumn{2}{|c|}{ Restauration hors domicile } & \multicolumn{2}{|c|}{ Commerçant détaillant } & \multicolumn{2}{|c|}{ Vente directe } & \multirow[b]{2}{*}{ GMS } \\
\hline & $\begin{array}{l}\text { Restauration } \\
\text { commerciale }\end{array}$ & $\begin{array}{c}\text { Restauration } \\
\text { collective }\end{array}$ & Non-fermier & Fermier & $\begin{array}{l}\text { Marchés de } \\
\text { plein vent }\end{array}$ & $\begin{array}{c}\text { Autres (Paniers, } \\
\text { VET, etc.) }\end{array}$ & \\
\hline \multirow{2}{*}{ A } & 0 & 23 & 0 & 0 & 0 & 7 & \multirow{2}{*}{70} \\
\hline & \multicolumn{2}{|c|}{23} & \multicolumn{2}{|c|}{0} & \multicolumn{2}{|c|}{7} & \\
\hline \multirow{2}{*}{ B } & 30 & 13 & 16 & 11 & 0 & 23 & \\
\hline & \multicolumn{2}{|c|}{$\frac{1}{43}$} & \multicolumn{2}{|c|}{$\frac{1}{27}$} & \multicolumn{2}{|c|}{23} & 1 \\
\hline \multirow{2}{*}{ c } & 0 & 7 & 27 & 26 & 7 & 7 & 26 \\
\hline & \multicolumn{2}{|c|}{7} & \multicolumn{2}{|c|}{53} & \multicolumn{2}{|c|}{14} & 26 \\
\hline \multirow{2}{*}{ D } & 10 & 22 & 13 & 16 & 0,5 & 0,5 & 38 \\
\hline & \multicolumn{2}{|c|}{32} & \multicolumn{2}{|c|}{29} & \multicolumn{2}{|c|}{1} & 38 \\
\hline \multirow{2}{*}{ E } & 87 & 13 & 0 & 0 & 0 & 0 & \\
\hline & \multicolumn{2}{|c|}{100} & & & & & 0 \\
\hline \multirow{2}{*}{$\mathrm{F}$} & 19 & 4 & 19 & 12 & 30 & 16 & \multirow{2}{*}{0} \\
\hline & \multicolumn{2}{|c|}{23} & \multicolumn{2}{|c|}{31} & \multicolumn{2}{|c|}{46} & \\
\hline
\end{tabular}

